\title{
Cumes. Recherches archéologiques dans la nécropole de la Porte médiane.
}

Campagnes 2017-2020

Jean-Pierre Brun, Priscilla Munzi, Marina Covolan, Elisa Conca, Saverio De Rosa, Barbara Del Mastro, Bastien Lemaire, Marcella Leone et Géraldine Sachau-Carcel

\section{OpenEdition}

Journals

Édition électronique

URL : https://journals.openedition.org/baefe/2166

DOI : $10.4000 /$ baefe. 2166

ISSN : 2732-687X

Éditeur

ResEFE

Référence électronique

Jean-Pierre Brun, Priscilla Munzi, Marina Covolan, Elisa Conca, Saverio De Rosa, Barbara Del Mastro, Bastien Lemaire, Marcella Leone et Géraldine Sachau-Carcel, « Cumes. Recherches archéologiques dans la nécropole de la Porte médiane. » [notice archéologique], Bulletin archéologique des Écoles françaises à l'étranger [En ligne], Italie, mis en ligne le 22 mai 2021, consulté le 24 mai 2021. URL: http://journals.openedition.org/baefe/2166 ; DOI : https://doi.org/10.4000/baefe.2166

Ce document a été généré automatiquement le 24 mai 2021.

\section{cc) (†)}

Le Bulletin archéologique des Écoles françaises à l'étranger est mise à disposition selon les termes de la Licence Creative Commons Attribution - Pas d'Utilisation Commerciale - Pas de Modification 4.0 International. 


\section{Cumes. Recherches archéologiques dans la nécropole de la Porte médiane.}

Campagnes 2017-2020

Jean-Pierre Brun, Priscilla Munzi, Marina Covolan, Elisa Conca, Saverio De Rosa, Barbara Del Mastro, Bastien Lemaire, Marcella Leone et Géraldine Sachau-Carcel

\section{NOTE DE L'ÉDITEUR}

La fouille de la nécropole de la Porte médiane de Cumes fait partie du programme de recherche « Aux marges de la ville de Cumes » porté par le Centre Jean Bérard (USR 3133, CNRS-EFR) avec le soutien du Parco archeologico dei Campi Flegrei et du Collège de France.

\section{NOTE DE L'AUTEUR}

Date précise de l'opération : 22 mai-30 juin 2017, 21 mai-29 juin 2018, 20 mai-28 juin 2019, 22 juin-03 juillet 2020.

Autorité nationale présente : Parco archeologico dei Campi Flegrei - MiC Numéro de mission : Concession MIBACT DGABAP Prot. n. 0011900 du 03/05/18 Composition de l'équipe de terrain : Les campagnes de fouille 2017-2019 ont réuni une équipe composée de professionnels, doctorants et étudiants : Obab Aldbiyat (Université Lyon), Olivier Afonsi (Université Grenoble Alpes), Simon Azema (archéologue indépendant), Jean-Pierre Brun (Collège de France), Guilhem Chapelin (CNRS, Centre Jean Bérard, USR 3133, CNRS-EFR), Elisa Conca (archéologue indépendante), Marina Covolan (Università degli studi di Salerno), Saverio De Rosa (archéologue indépendant), Barbara Del Mastro (archéologue indépendante), Macarena 
Enriquez de Salamanca (Université de Tours), Marianne Esso Camargo (Université Paul Valéry Montpellier 3), Anthony Gillot (Université de Bourgogne), Allan Girot (Université de Bourgogne), Pauline Gutzwiller (Université de Haute-Alsace), Noémie Ledouble (archéologue indépendante), Bastien Lemaire (Université Paul-Valéry Montpellier 3, UMR 5140 ASM), Marcella Leone (CNRS, Centre Jean Bérard, USR 3133, CNRS-EFR), Flore Lerosier (Université de Tours), Stéphanie Mailleur (University of Southampton), Céline Maréchal (Université de Bourgogne), Rodolphe Martinez (Université Paul Valéry Montpellier 3), Rafael Monpean (Universidade de São Paulo), Priscilla Munzi (CNRS, Centre Jean Bérard, USR 3133 CNRS-EFR), Dorothée Neyme (Centre Jean Bérard, USR 3133 CNRS-EFR), Marie Pawlowicz, (Université d'AixMarseille), Caua Pitta Parente (Universidade de São Paulo), Francisco Bruno Resella (Universidade de São Paulo), Camille Roche (Université Paris 1 Panthéon-Sorbonne), Pauline Roussey (Université de Bourgogne), Géraldine Sachau-Carcel (Université PaulValéry Montpellier 3, UMR 5140 ASM), Téo Shillinger-Geis (Université de Haute-Alsace), Anna Susini (archéologue indépendante), Clara Tourniaire (Université d'Aix-Marseille), Anissa Yelles (Université Paris 1 Panthéon-Sorbonne), Corinne Vallar (archéologue indépendante), Ophélie Vauxion (Centre Jean Bérard, USR 3133, CNRS-EFR), Alessia Vittorioso (restauratrice indépendante), Jordan Voillot (Université de Bourgogne).

Partenariats institutionnels : CJB, Collège de France

Établissement éditeur : CJB

Établissements porteurs de l'opération : CJB

Remerciements : Nous tenons à remercier de leur aide et appui le dott. Fabio Pagano, directeur du Parco, le dott. Filippo Demma, l'architecte Marida Salvadori, et la dott.ssa Marzia Del Villano, Cesare Giordano et Gennaro Carandente (Ufficio per i beni archeologici di Cuma). Les recherches à Cumes sont financées par le ministère de l'Europe et des Affaires étrangères (Mission archéologique "Italie du Sud »), le CNRS, l'École française de Rome et la Fondation du Collège de France. Les opérations ont en outre bénéficié d'une dotation au titre du mécénat de la Fondation ARPAMED (Archéologie et Patrimoine en Méditerranée) en 2019 et en 2020.

Données scientifiques produites :

https://centrejeanberard.cnrs.fr/spip.php?article34\&lang=fr

Chroniques de l'EFR :

https://journals.openedition.org/cefr/990

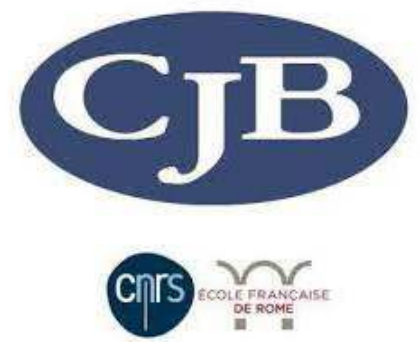




\section{Introduction}

En 2001, le Centre Jean Bérard a lancé un programme de recherche pour étudier la nécropole de Cumes qui s'étend à l'extérieur des fortifications septentrionales (fig. 1-2).

Fig. 1. Le site de Cumes et au premier plan la nécropole de la Porte médiane.

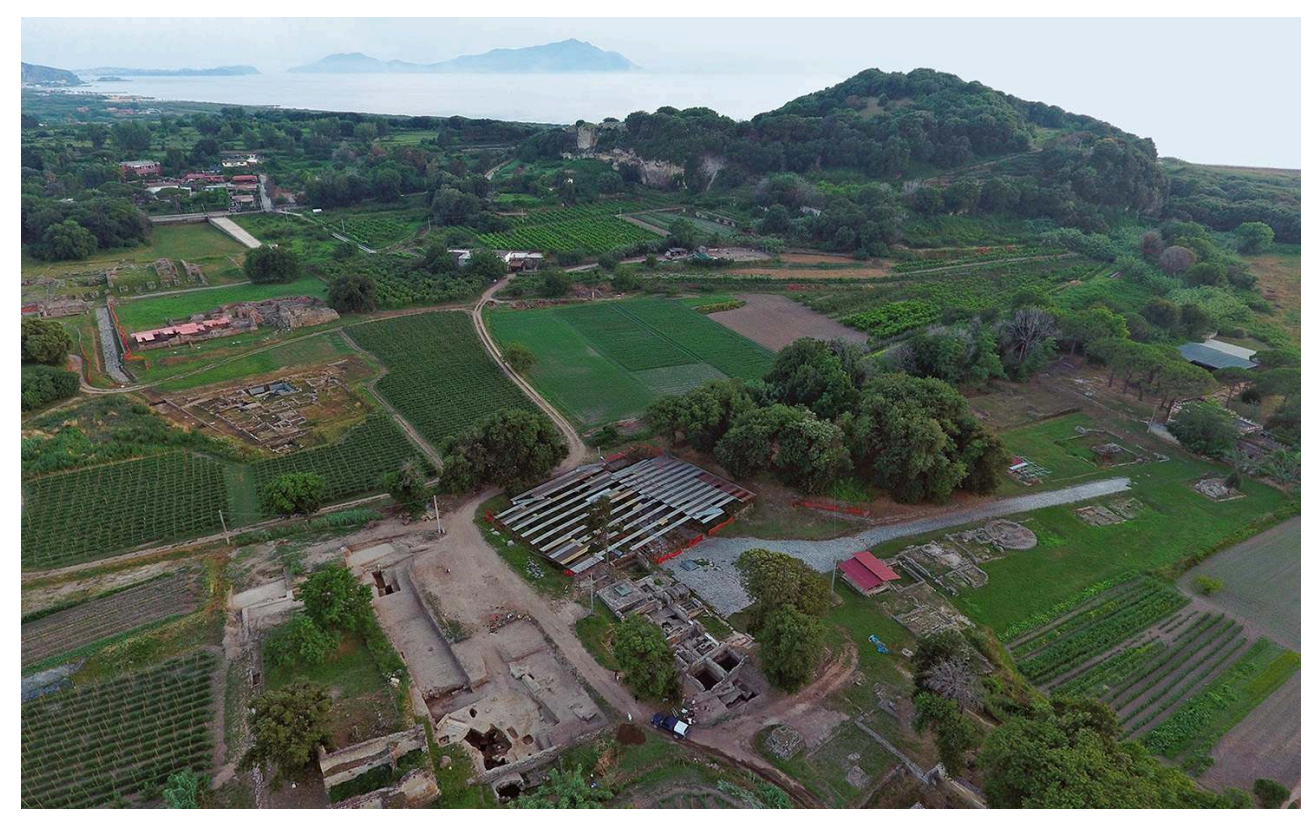

E. Botte, CNRS, CCJ / @ CC BY-NC-ND. 
Fig. 2. Plan la nécropole de la Porte médiane.

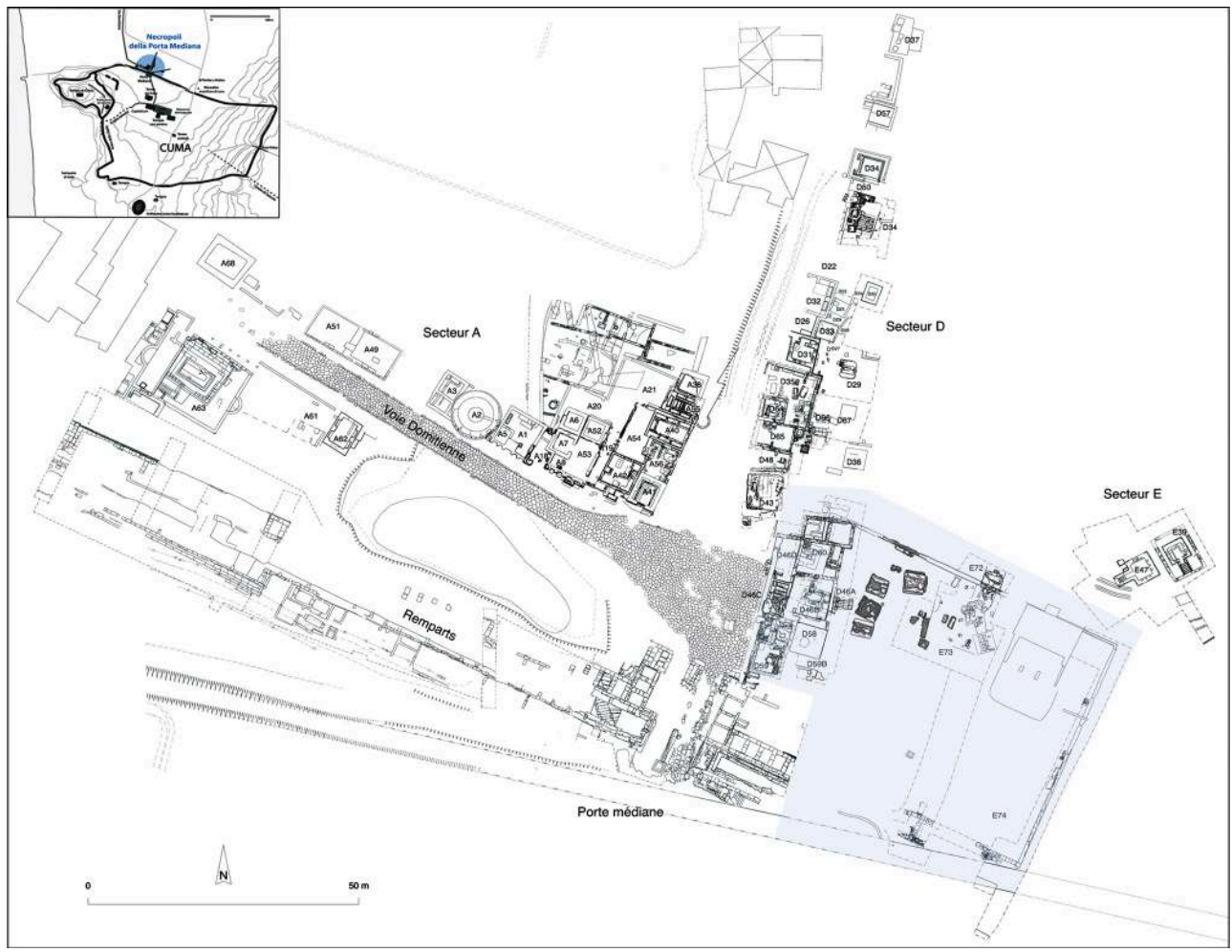

Réal. G. Chapelin, CJB, CNRS-EFR / @ CC BY-NC-ND.

Les recherches menées entre 2017 et 2020 se sont concentrées sur la zone située immédiatement au nord-est de la Porte médiane, un secteur qui a connu d'importantes phases de transformation, à la fois à la fin de l'époque hellénistique $\left(\mathrm{II}^{\mathrm{e}} \mathrm{I}^{\mathrm{er}}\right.$ siècle av. J.-C.), lorsque le fossé défensif situé à l'extérieur des murs est comblé1, et à l'époque flavienne, lorsque la via Domitiana est construite et que la zone de la Porte est monumentalisée ${ }^{2}$. Dans les deux cas, les réorganisations de l'espace situé immédiatement à l'extérieur des murailles ont également impliqué la nécropole. Ces recherches ont donné lieu à plusieurs comptes rendus dans la Chronique des activités archéologiques de l'École française de Rome, en particulier dans celle de 2019 où nous avons exposé les résultats obtenus pour les phases d'époque impériale 3 .

Les fouilles de ces dernières années ont comporté une série de sondages stratigraphiques qui ont permis d'étudier les niveaux de la nécropole hellénistique, oblitérés ou incorporés dans l'organisation du grand complexe impérial, et présentés ici (fig. 3-4) 
Fig. 3. Orthophotographie de la nécropole de la Porte médiane, juin 2019.

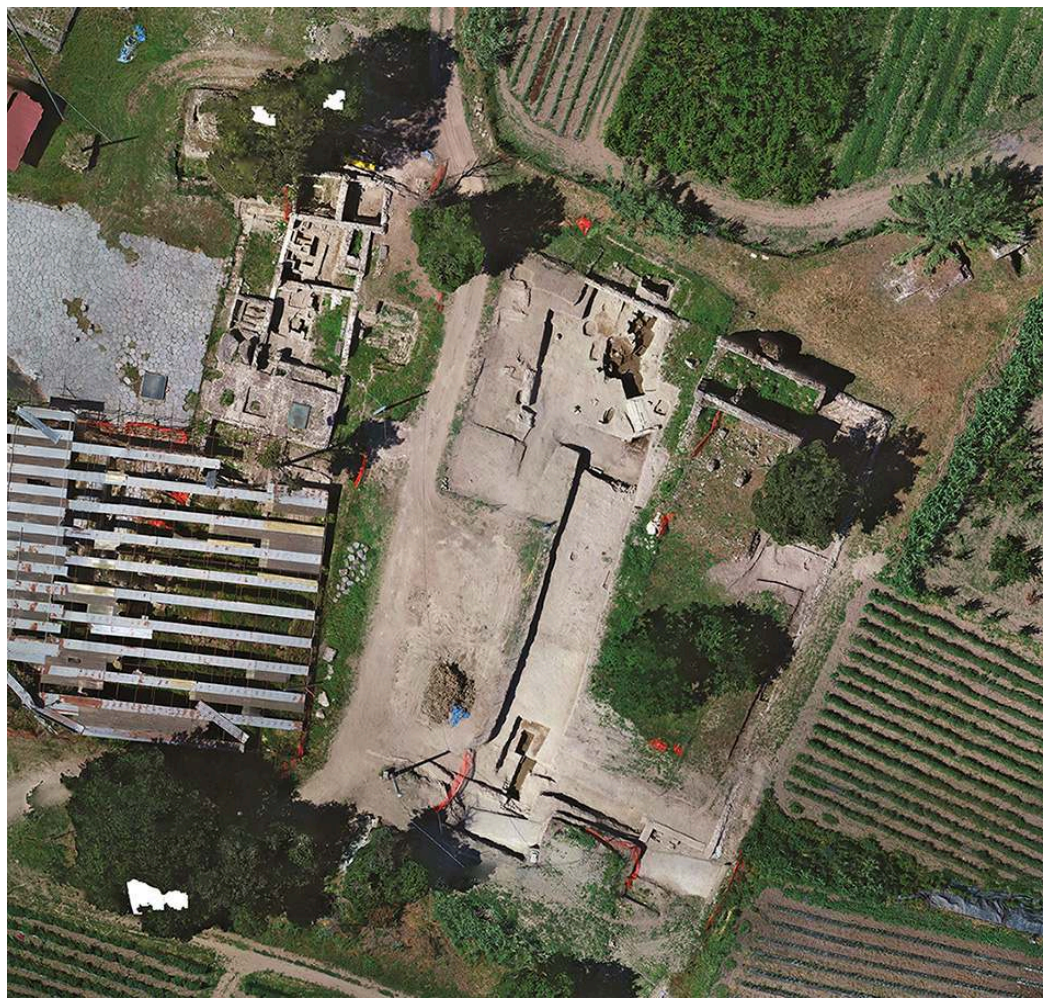

Réal. R. Catuogno, DiStar UniNa, M. Facchini, MLab UniNa, M. Giglio, UniOr / @ CC BY-NC-ND.

Fig. 4. Plan du secteur de nécropole situé au nord-est de la Porte médiane.

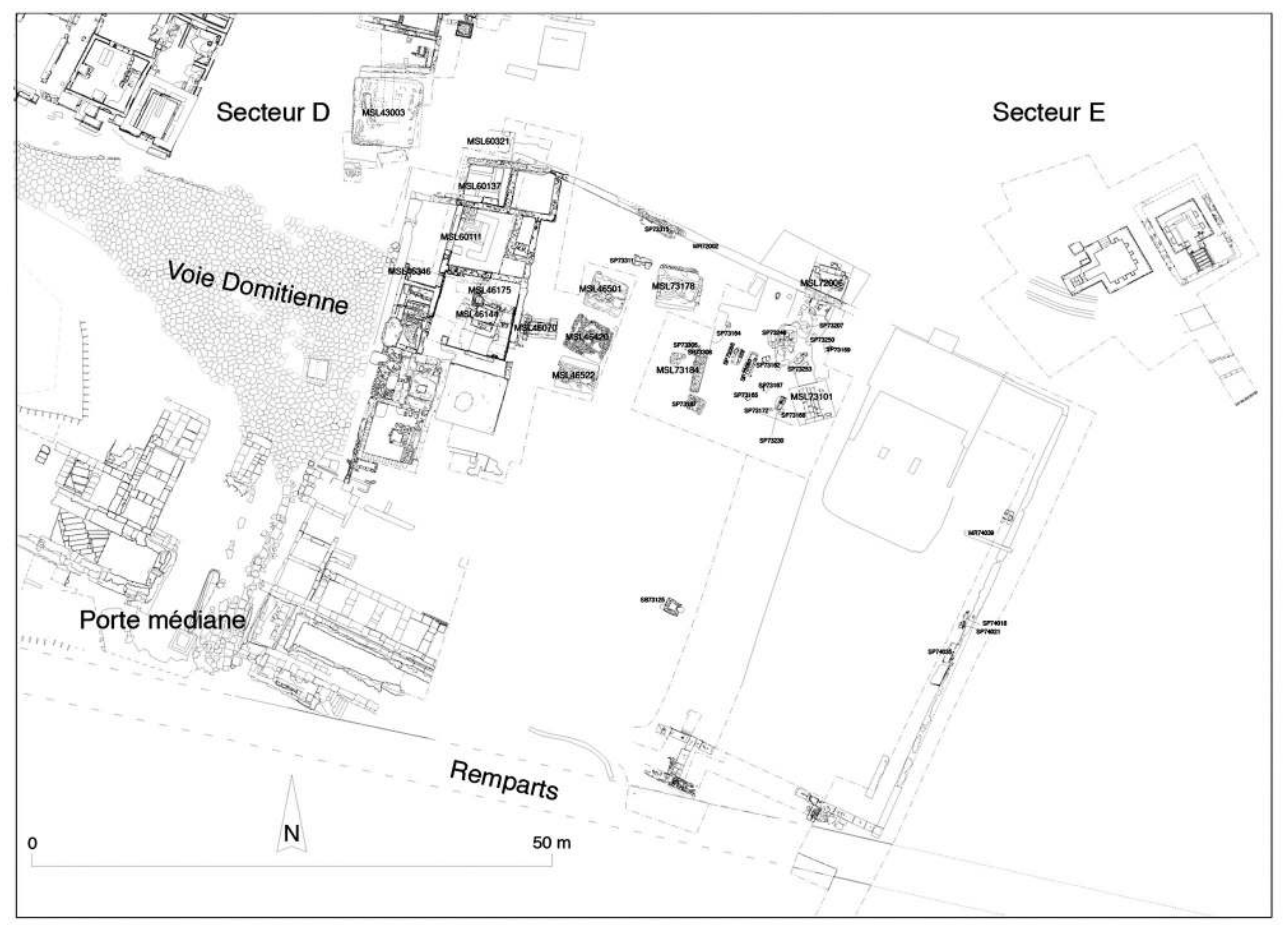

Réal. G. Chapelin, M. Covolan, CJB, CNRS-EFR / @ CC BY-NC-ND 


\section{La nécropole aux III ${ }^{\mathrm{e}} \mathrm{II}^{\mathrm{e}}$ siècles av. J.-C.}

Les campagnes de fouilles menées entre 2017 et 2019, dans la zone située à proximité de la Masseria de Matteo Scotto d'Aniello le «Procidano » ont permis de dégager une série de tombes à inhumation d'époque hellénistique dont l'architecture était déjà bien connue grâce aux fouilles du XIX ${ }^{e}$ siècle ${ }^{5}$.

Un sondage sous le pavement du tombeau à chambre hypogée à voûte en berceau MSL72006, situé sous le mur d'enceinte nord de la terrasse d'époque flavienne, a révélé la présence de deux sépultures antérieures (fig. 5).

Fig. 5. Les sépultures SP72042 et SP72046.

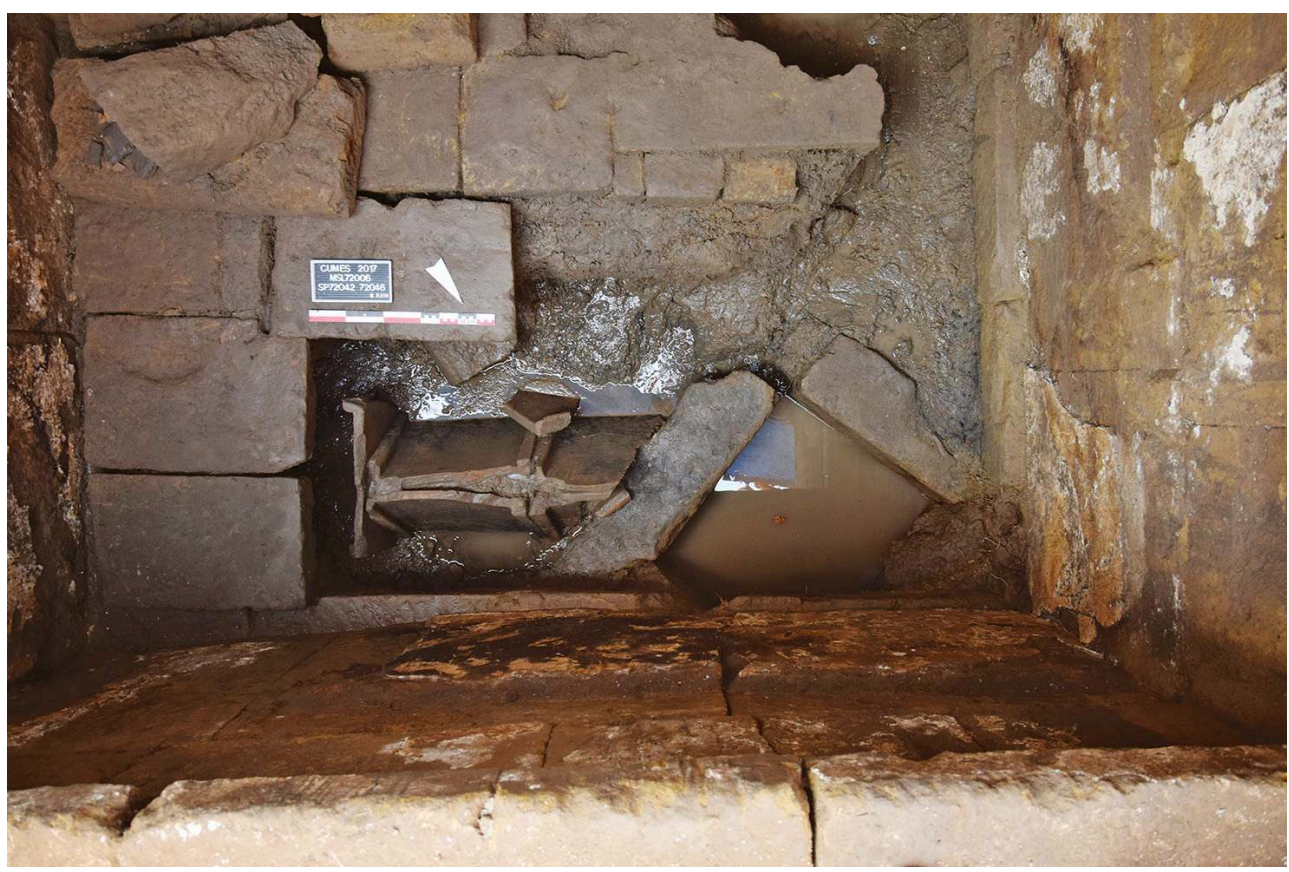

Archives CJB, CNRS-EFR / (C) CC BY-NC-ND

6 La plus ancienne est une tombe à inhumation en fosse avec couverture de tuiles en bâtière, SP72042, tombe d'un enfant déposé à l'intérieur d'un kalypter décoré de bandes diagonales qui servait de fond ; deux tuiles de type tegula protégeaient la sépulture sur chacun des longs côtés et une sur les côtés courts ; la sépulture était orientée est-ouest, avec la tête du défunt placée à l'est $(0,90 \times 0,55 \mathrm{~m})$. La présence de la nappe phréatique a rendu difficiles les opérations de fouille et l'identification des creusements liés à la mise en place de la tombe. Malgré cela, le kalypter sur lequel était placé le corps a pu être prélevé sans difficulté et la fouille a été effectuée en laboratoire. L'affaissement des côtes, les différents mouvements des corps vertébraux et de la mandibule indiquent une décomposition en espace vide.

7 Cette tombe « alla cappuccina » SP72042 a été partiellement détruite par l'aménagement d'une tombe en caisson ( $a$ cassa piana »), SP72046, malheureusement déjà pillée dans l'Antiquité. La structure $(1,70 \mathrm{x} 0,70 \mathrm{~m})$, d'orientation nord-est/sud-ouest, était composée de quatre dalles de tuf et n'avait pas de fond aménagé. À l'intérieur, les restes d'un individu adulte de sexe masculin ont été retrouvés. Il reposait sur le dos, les membres inférieurs en extension, le pied gauche sur le pied droit. Seule la partie 
inférieure du corps, à partir du bassin, a été prélevée ainsi que le radius et l'ulna gauches et la main droite, la partie supérieure étant absente à cause du pillage de cette tombe. Aucune pathologie osseuse n'a été observée.

8 En l'absence de mobilier funéraire, la datation est fournie par la stratigraphie : les sépultures sont placées chronologiquement entre la fin du $\mathrm{VI}^{\mathrm{e}}$ et le $\mathrm{II}^{\mathrm{e}}$ siècle av. J.-C.

Une intervention réalisée en 2019 dans la même zone, immédiatement au sud du mur d'enceinte nord de la terrasse, mit au jour un tunnel profond et long (CN73043), crée par des fouilleurs professionnels au XIXe siècle. Sa fouille a permis la découverte de quatre sépultures, déjà pillées, datant de la fin de l'époque hellénistique, dont trois sont à caisson et une est de type à fosse simple, et d'une stratigraphie complexe clairement visible dans les parois de la coupe (fig. 6).

Fig. 6. Le tunnel CN73043 et les sépultures à caisson SP73250, SP73253 et SP73246.

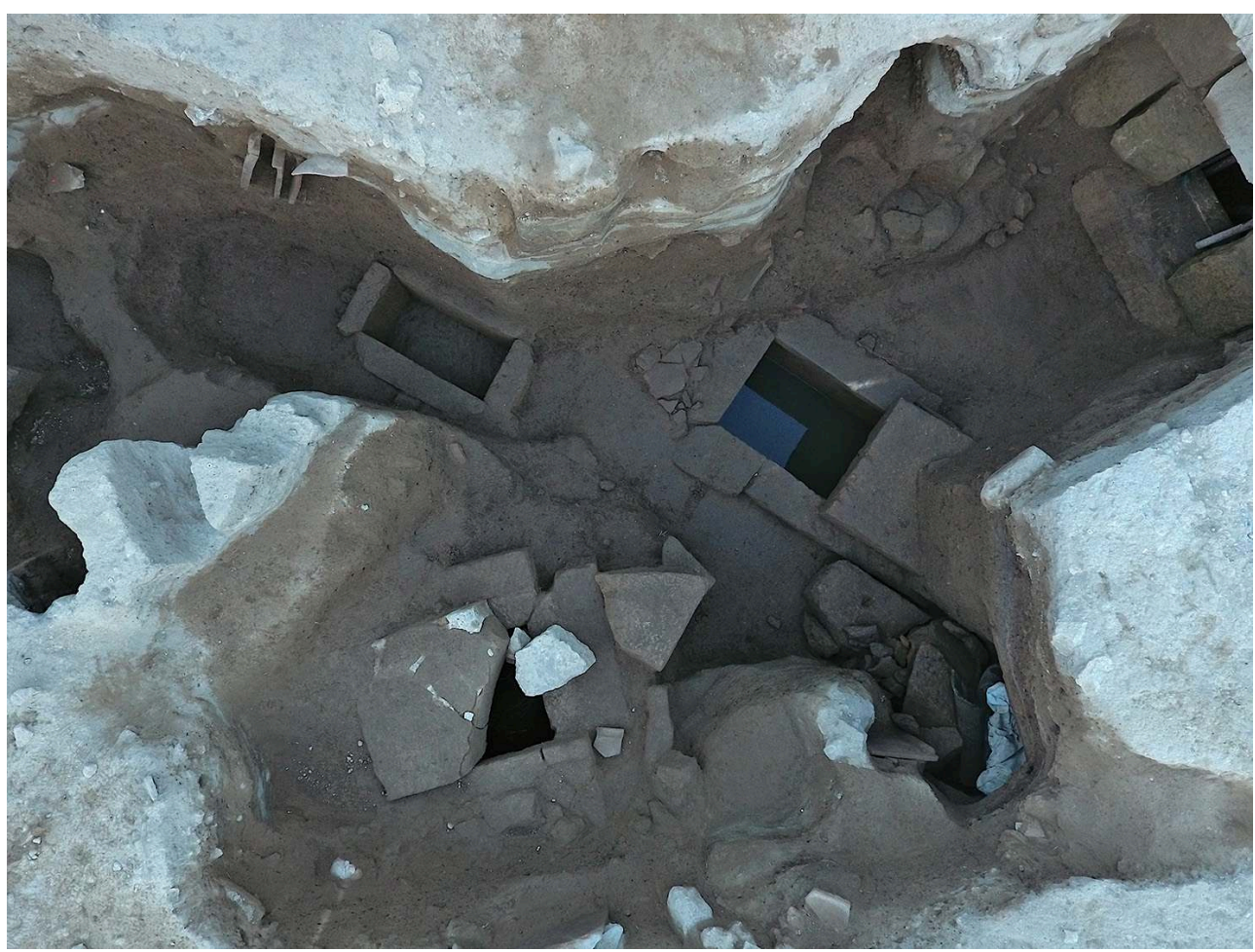

Archives CJB, CNRS-EFR / (C CC BY-NC-ND.

La première, SP73250, est une tombe en caisson. La structure est composée de quatre dalles de tuf jaune, placées de chant, l'une contre l'autre de manière à créer un espace interne rectangulaire d'environ $0,45 \mathrm{~m}$ de large, de $0,95 \mathrm{~m}$ de long et environ de $0,45 \mathrm{~m}$ de hauteur. Le fond de la tombe était également recouvert d'une dalle de tuf. Elle est entièrement perturbée et les restes squelettiques sont fragmentés. Seuls subsistent la mandibule qui a permis de déterminer l'âge de l'individu, l'humérus droit et une partie du gauche, le sacrum et l'extrémité proximale des fémurs. Le sujet inhumé est un sujet immature âgé entre 3 et 6 ans.

11 Les deux autres sépultures, SP73253 et SP73246, avec caisson de dalles de tuf, sont du type «a connola» ou " a culla», caractérisé par la présence, entre les parois et le couvercle, d'un bandeau mouluré, également fait de blocs. Stevens, dans son tableau 
typologique publié en 1883, en reproduit plusieurs variantes selon la taille des tombes ou la présence de niches sur les côtés longs ${ }^{6}$.

La tombe SP73253 semble la moins remaniée; elle a conservé la partie supérieure du squelette d'un sujet adulte placé sur le dos, tête vers le sud. La structure de la tombe ( $2 \times 0,90 \mathrm{~m})$, assez bien conservée, est composée d'un fond de sept dalles de tuf à plat, sur lequel reposent sept grosses dalles, placées de chant, sur tout le périmètre, elles-mêmes surmontées de six blocs, épais d'environ $0,40 \mathrm{~m}$ et posés à plat; ils sont moulurés, avec un bandeau supérieur droit et un bandeau inférieur oblique ${ }^{7}$. Deux grosses dalles, d'un peu plus de $0,90 \mathrm{~m}$ de côté et d'environ $0,30 \mathrm{~m}$ d'épaisseur, scellaient la sépulture. À l'intérieur du caisson funéraire, ont été découverts en position primaire, laissés par les fouilleurs modernes, deux unguentaria en céramique, appartenant au type fusiforme à anses posées horizontalement sur l'épaule (type Camilli B.51.2), typologie courante dans les mobiliers funéraires à Tarente entre la fin du III ${ }^{e}$ et la première moitié du II ${ }^{\mathrm{e}}$ siècle av. J.-C.

13 La tombe "a connola » SP73246, plus récente, était déjà complètement perturbée et aucun vestige n'a été récupéré en position primaire. Le tamisage du remplissage a livré quelques éléments métalliques, dont une pièce de monnaie et des fragments de plaquette en bronze, dont l'attribution à la sépulture est incertaine ${ }^{8}$. En partant du fond, le lit de déposition du défunt était constitué de douze de dalles de tuf côte à côte, sur lesquelles posaient six grosses dalles de périmètre et, entre celles-ci et la couverture, une rangée de blocs dans lesquels était découpée une bande moulée (2,10 x $1,05 \mathrm{~m})^{9}$. Trois dalles de tuf de dimensions et d'épaisseurs différentes, fermaient la tombe; seule la plus à l'est avait été arrachée par les fouilleurs du XIX siècle pour accéder à la chambre funéraire.

14 La tombe SP73207, la plus récente des quatre, à fosse simple, est celle d'un individu immature, dont le squelette, trouvé incomplet, était placé sur le dos, crâne au nordouest. Seule la partie supérieure du corps est conservée. Les différents ossements effondrés (crâne, côtes) et déplacés (vertèbres thoraciques) attestent une décomposition du cadavre dans un espace vide; la fosse était donc recouverte par un élément en matériau périssable tel qu'une planche. Le creusement de la fosse était presque rectangulaire avec des parois un peu obliques et au profil légèrement concave. Un clou en fer et une monnaie de bronze ont été découverts au contact de l'humérus gauche, entre les côtes et la scapula gauches ${ }^{10}$. Le sujet immature était âgé entre 10 et 14 ans d'après le stade de fusion des différentes épiphyses des membres supérieurs.

La sépulture a été partiellement entaillée par la fouille du dromos de l'hypogée MSL72006 et par celle du tunnel moderne.

Les quatre sépultures, sur la base des quelques objets funéraires découverts et de la stratigraphie, peuvent être datés entre le $\mathrm{III}^{\mathrm{e}}$ et le $\mathrm{II}^{\mathrm{e}}$ siècle av. J.-C.

La plupart des tombes des $\mathrm{IV}^{\mathrm{e}}$ et $\mathrm{III}^{\mathrm{e}}$ siècles a été découverte au XIX ${ }^{\mathrm{e}}$ siècle près des murs nord-est de la ville, le long de la voie qui sortait de la Porte orientale. Les recherches récentes montrent que la nécropole samnite se développait vers l'ouest jusqu'à l'axe nord-sud qui sortait de la Porte médiane et vers le sud près des fortifications septentrionales ${ }^{11}$. Les anciennes parcelles funéraires continuent à être utilisées, «dans une continuité partielle et dans le respect de l'ordre antérieur » ${ }^{12}$. La réorganisation des espaces s'accompagne d'un changement net dans les rituels funéraires, le mobilier et la typologie de tombes ${ }^{13}$. Les sépultures qui viennent d'être décrites confirment la présence de niveaux de nécropoles hellénistiques dans ce 
secteur périphérique de Cumes où se trouve une concentration de sépultures à inhumation.

\section{La nécropole entre le milieu du $\mathrm{Il}^{\mathrm{e}}$ et le premier quart du ler siècle av. J.-C.}

Entre le dernier quart du $\mathrm{II}^{\mathrm{e}}$ siècle et les premières décennies du I ${ }^{\mathrm{er}}$ siècle av. J.-C., la zone à proximité de la Porte, à l'est de la voie nord-sud (D), est occupée par une série de tombes hypogées à voûtes en berceau, construites en gros blocs de tuf. Autour de ces monuments, destinés à être utilisés pour des inhumations, de nombreuses tombes individuelles à crémation sont disposées dans des fosses sous des stèles ou cippi en tuf portant parfois des inscriptions en osque ou en latin ${ }^{14}$.

19 Les fouilles, menées entre les remparts et le mur nord de la terrasse d'époque flavienne, ont mis au jour, sous les couches de remblai utilisées pour l'aménagement de celle-ci, deux tombeaux à chambre hypogée destinés à accueillir des inhumations (MSL72006 et MSL73101) et une série de sépultures à crémation sous cippe.

\section{Le tombeau à chambre hypogée avec voûte en berceau MSL72006}

L'édifice funéraire MSL72006, dont la structure porteuse est faite de gros blocs de tuf équarris assemblés à sec, est du type à chambre hypogée, avec une façade imposante et une rampe à gradins qui permettait d'accéder à la structure par le sud (fig. 7). L'intérieur est de plan quadrangulaire (environ $3,50 \times 3,68 \mathrm{~m}$; hauteur $3,50 \mathrm{~m}$ ), avec une voûte en berceau et des murs construits de trois rangées de blocs superposés, dont l'un constitue la corniche faite d'une moulure à cyma reversa. Les parois intérieures du tombeau sont recouvertes d'une couche d'enduit blanc, et la voûte, en blocs de tuf, débute au-dessus de la corniche. Cette voûte, large de 2,70 m, décharge son poids sur les murs est et ouest et se développe sur une longueur de 2,80 pour 1,20 m de hauteur sous la clé. L'accès depuis le dromos est aménagé dans le mur sud de la chambre; on y accède par une entrée à sommet semi-circulaire, placée en position excentrée, fermée par un monolithe de tuf au moment de la fouille. Cette ouverture est marquée par un seuil qui est situé au même niveau que le pavement réalisé en blocs de tuf disposés côte à côte, à sec. 
Fig. 7. Le tombeau à chambre hypogée MSL72006.

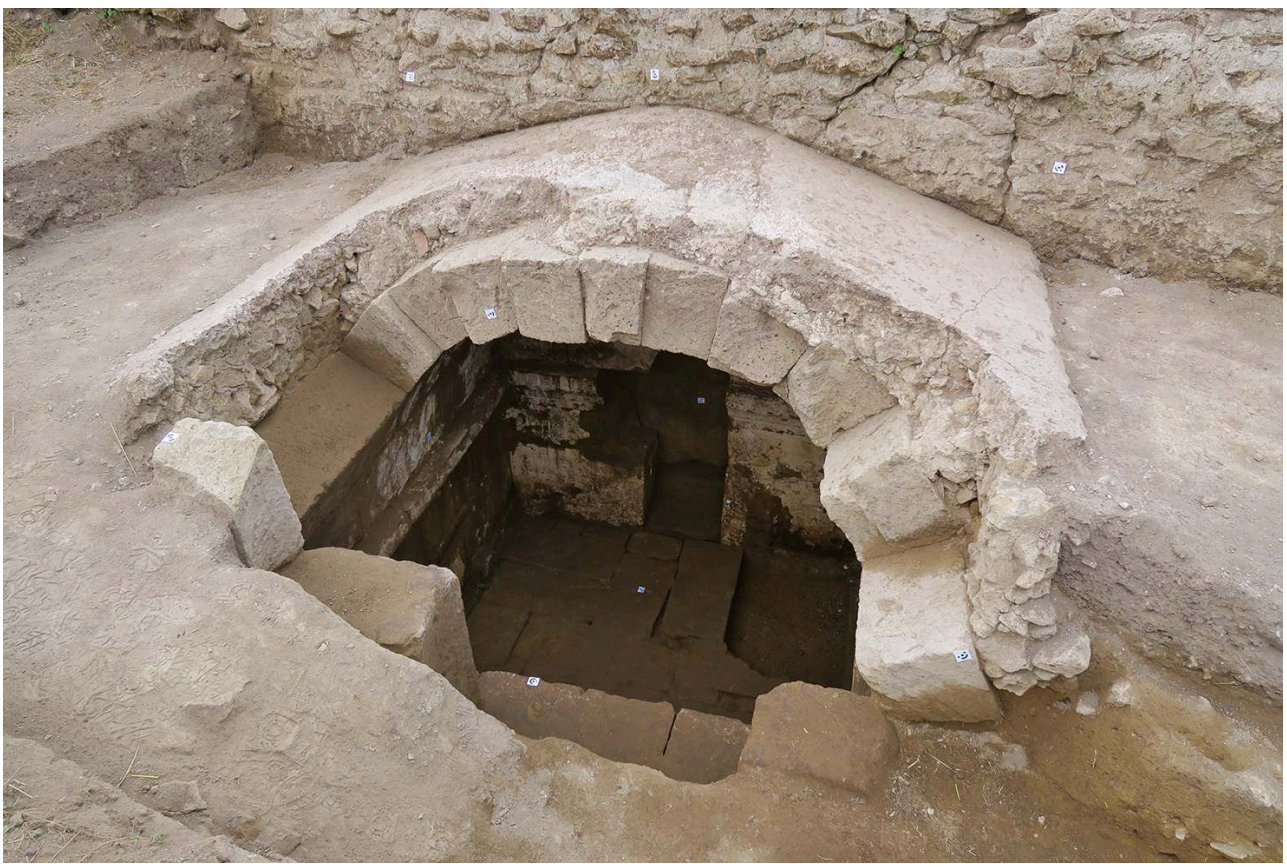

Archives CJB, CNRS-EFR / @ CC BY-NC-ND.

21 La répartition de l'espace funéraire, qui comprenait trois inhumations en caisson de dalles de tuf (SP72018, SP72019 et SP72020), a été reconnue grâce aux empreintes visibles sur les murs est, ouest et nord, car les structures ont été entièrement spoliées. Ces empreintes sont les négatifs des couvertures lithiques des lits funéraires. Les trois sépultures utilisent comme fond le sol en dalles de tuf de la chambre funéraire. La présence de traces de mortier près des négatifs suggère que les caissons étaient entièrement enduits. Ils étaient occupés par des inhumations qui prévoyaient la déposition du défunt et du mobilier au-dessus d'une couche de sable marin.

Bien que la tombe ait été retrouvée altérée et pillée, à la fois durant l'Antiquité et à l'époque moderne, la fouille a permis de récupérer partiellement les restes osseux des individus déposés et certains éléments du mobilier funéraire. Dans l'un des dépôts, une substance organique brune, adhérant largement aux restes osseux du défunt, a été trouvée. Il pourrait s'agir des restes d'une planche de bois probablement utilisée pour transporter ou caler le défunt.

Au moment de sa découverte, la chambre funéraire était remplie jusqu'au sommet de la voûte par une importante quantité de matériel céramique hétérogène, mélangé à de la terre et à des gravats. Le dégagement de ce remblai a mis en évidence une couche plus compacte à l'interface de laquelle ont été identifiés de nombreux artefacts attribuables au mobilier associé aux sépultures. Bien que les caissons funéraires aient été enlevés, il a été possible d'isoler plusieurs lots d'objets localisés dans les espaces réservés aux caissons, au centre et dans l'angle nord-est de la pièce (fig. 8). 
Fig. 8. Mobilier funéraire du tombeau à chambre hypogée MSL72006.

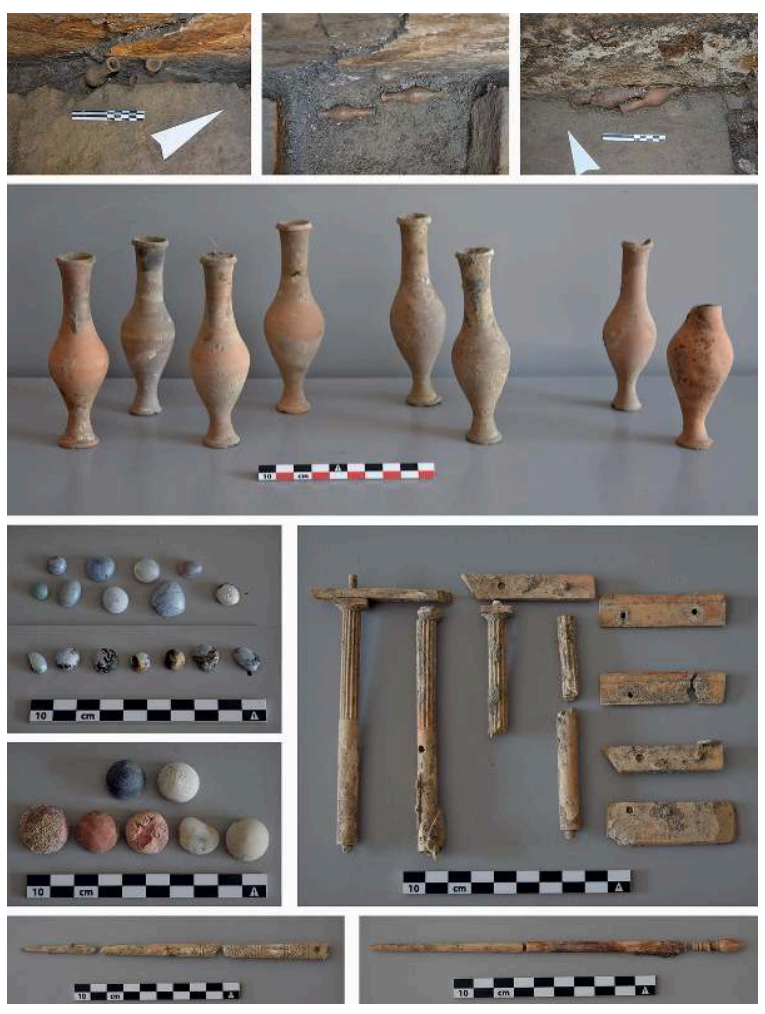

Archives CJB, CNRS-EFR / @ CC BY-NC-ND.

La sépulture le long du mur est de la chambre a restitué un petit groupe de matériel vraisemblablement disposé près de la tête du défunt. Il s'agit de fragments de céramique, dont le bord d'un unguentarium fusiforme, une petite tige de fer et des fragments d'un miroir circulaire en argent.

La quantité de matériel trouvé dans le second caisson funéraire - dont l'empreinte s'étendait le long du mur ouest de la chambre - est plus abondante. Au total, 23 objets ont été collectés, dont la plupart ont été trouvés dans sa partie sud-ouest. Parmi eux, il y a 16 pions de jeu en verre et en pierre, un galet blanc, un peigne en os à double denture, une applique circulaire en bronze et un petit clou en fer encore fixé à du bois. Ces derniers objets appartenaient probablement à une boîte ou à un petit contenant en bois. L'interprétation des autres objets métalliques découverts est plus problématique. Ce sont des éléments en fer: une tige à section ovale, une deuxième tige et une plaquette, faisant vraisemblablement partie de l'instrumentum.

La zone occupée par la sépulture nord est la plus touchée par l'action violente des clandestins, et le matériel recueilli est d'attribution incertaine et peut-être pertinent aux autres dépositions. 21 objets ont été collectés, auxquels il faut ajouter les unguentaria trouvés coincés entre les dalles du sol et les murs de la chambre funéraire. La plupart sont en métal, fer et bronze. Parmi les objets en fer, on trouve un fragment de ligula d'un strigile et plusieurs fragments de tiges à section circulaire dont les surfaces semblent avoir été en contact avec du bois. Il n'est pas exclu que ces dernières aient fait partie d'un coffre en bois ou d'un lit funéraire, auquel on pourrait également attribuer un gros clou à tête ronde, un clou en $\mathrm{L}$ et une applique en os qui a encore son clou de fixation. Parmi les objets personnels, on trouve quelques fragments d'au moins 
deux miroirs à disque, la partie supérieure d'une épingle en os, alors qu'au domaine social sont à attribuer quelques pions de jeu en verre et en pierre.

Aux objets qui viennent d'être décrits, il faut ajouter huit unguentaria fusiformes en céramique (cum-UNG A.III / équivalent de Camilli B.13), trouvés en deux lots entre les dalles du sol et les parois ouest et nord.

Dans l'angle sud-est de la chambre funéraire, une accumulation de matériel a été mise au jour, probablement le résultat de l'action des clandestins. Parmi ce matériel se trouvaient un unguentarium fusiforme (CUM-UNG A.III), une amphorette en céramique commune, une coupelle à paroi fine avec un fond plat et de petites anses placées horizontalement sur le bord et un alabastron en albâtre (Colivicchi, classe II, forme 1.3). Sur le même niveau ont également été collectés 18 pions en verre de forme et de couleur différentes, un pion en pierre et un galet de forme régulière, peut-être utilisé également comme un jeton de jeu. Les objets métalliques ne sont pas moins nombreux : des fragments de ligula et un anneau de suspension d'un strigile, tous deux en fer; un fragment d'une paire de forces et plusieurs petits éléments en bronze tels que des appliques circulaires bombées, des anneaux mobiles, des poignées et des clous en fer de différentes formes et dimensions, à rattacher probablement à des objets en bois dont il ne reste rien.

Les nombreux éléments en os, dont la plupart ont été trouvés dans le deuxième niveau de remblai, peuvent également être associés à un coffret au moins. Il faut noter en particulier les quatre petites colonnes travaillées en ronde-bosse. Ce sont des colonnes à chapiteaux doriques dont seule la partie supérieure est cannelée tandis que la moitié inférieure est lisse. Entre une colonne et l'autre, il devait y avoir une balustrade ajourée avec un motif de réticulé et le haut de la petite colonnade était couronné d'un entablement dentelé. Les nombreuses plaques d'os qui ont été recueillies devaient recouvrir les autres côtés du coffret et de son couvercle. Plus problématique est l'attribution des différents bandeaux fins et des éléments de charnières cylindriques en os à trou latéral, pouvant appartenir aussi bien à une boîte à miroirs qu'au coffret qui vient d'être décrit. En plus de tous ces éléments, un stylet en os et trois broches en fer ont également été retrouvés.

30 Tous ces objets trouvent d'amples comparaisons dans les contextes de la fin de l'époque républicaine et de l'époque augustéenne dans le centre et le sud de l'Italie et confirment une utilisation du monument entre le dernier quart du $\mathrm{II}^{\mathrm{e}}$ siècle et le premier quart du Ire siècle av. J.-C.

31 Le creusement de la fondation de ce dernier monument coupe la sédimentation précédente et il est probable que les travaux de terrassement liés à la construction du mausolée aient été réalisés au même moment que la rampe d'accès à gradins orientée nord/nord-ouest sud/sud-est, qui s'écarte légèrement de l'axe de la tombe. La chambre funéraire contenait trois sépultures à caisson; bien qu'il soit probable qu'il y ait eu au moins deux réouvertures du monument, à la suite de la déposition du premier défunt, la stratigraphie n'atteste qu'une seule d'entre elles.

Le couloir d'accès à l'hypogée menait, dans sa dernière phase, à l'axe routier en terre battue V073161, qui s'étendait à environ $5 \mathrm{~m}$ au sud de l'entrée du tombeau avec une orientation est/nord-est-ouest/sud-ouest (la route semble avoir un peu plus de 2,50 m de large). Dans la partie supérieure du mur ouest, juste devant la façade du monument, il y a un petit mur composé de quatre rangées de blocs de tuf irréguliers, dont la 
fonction est à mettre en lien avec les opérations de déplacement de l'imposante pierre monolithe de fermeture de la chambre funéraire.

La dernière phase d'utilisation du mausolée est marquée par une importante intervention de maintenance. La voûte en berceau de blocs de tuf est stabilisée grâce à la réalisation d'une autre couverture, faite d'éclats de tuf et de mortier, recouverte par une couche de mortier de tuileau, qui s'appuie sur deux murs latéraux en opus incertum/ quasi reticulatum avec des moellons irréguliers et des cubilia de grandes dimensions, grossièrement taillés en surface et disposés selon un alignement irrégulier.

L'abandon définitif du tombeau est marqué par l'arrêt de l'utilisation du dromos, qui est comblé par une terre riche de blocs de tuf jaune grossièrement taillés, de pierres, d'éclats de tuf, de fragments de briques, de fragments de marbre et d'agglomérés de chaux. Plus tard, à une période difficile à dater en raison de l'absence de mobilier funéraire, le remblai est creusé pour mettre en place une sépulture à fosse simple pour un sujet adulte (SP73051), déposé sur le dos et avec le crâne orienté vers le nord. Cette tombe est incomplète car perturbée par le percement du tunnel réalisé par les fouilleurs du XIX ${ }^{e}$ siècle.

\section{Le tombeau à chambre hypogée avec voûte en berceau MSL73101}

Dans les premières décennies $d u \mathrm{I}^{\text {er }}$ siècle $\mathrm{av}$.J.-C., à quelques mètres au sud du monument funéraire MSL72006, une nouvelle tombe à chambre hypogée avec voûte en berceau est construite (MSL73101) ${ }^{15}$. Orienté sud-est/nord-ouest, avec accès par le sud, le tombeau est construit avec des rangées régulières de blocs de tuf jaune, parallélépipédiques et de tailles variées, portant des signes de travail et posés à sec. Il possède une façade monumentale qui se développe en hauteur sur environ $4 \mathrm{~m}$ et dont le sommet est surélevé d'environ $1 \mathrm{~m}$ par rapport à la clé de voûte. L'hypogée était accessible par un dromos aux parois de terre dont seule la partie proche de l'entrée a été interceptée.

L'entrée rectangulaire, avec un linteau monobloc cintré, était fermée par un monolithe de tuf jaune, dont la face supérieure était recouverte d'une couche de mortier qui adhérait au mur de façade pour assurer une meilleure étanchéité. L'intérieur est de plan presque carré (environ $3 \mathrm{~m}$ de côté pour une hauteur maximale d'environ $3 \mathrm{~m}$ ), avec une corniche, en saillie et modelée à cyma reversa, courant sur les quatre côtés et sur laquelle est posée la voûte en berceau (fig. 9). 
Fig. 9. L'intérieur du tombeau à chambre hypogée MSL73101.

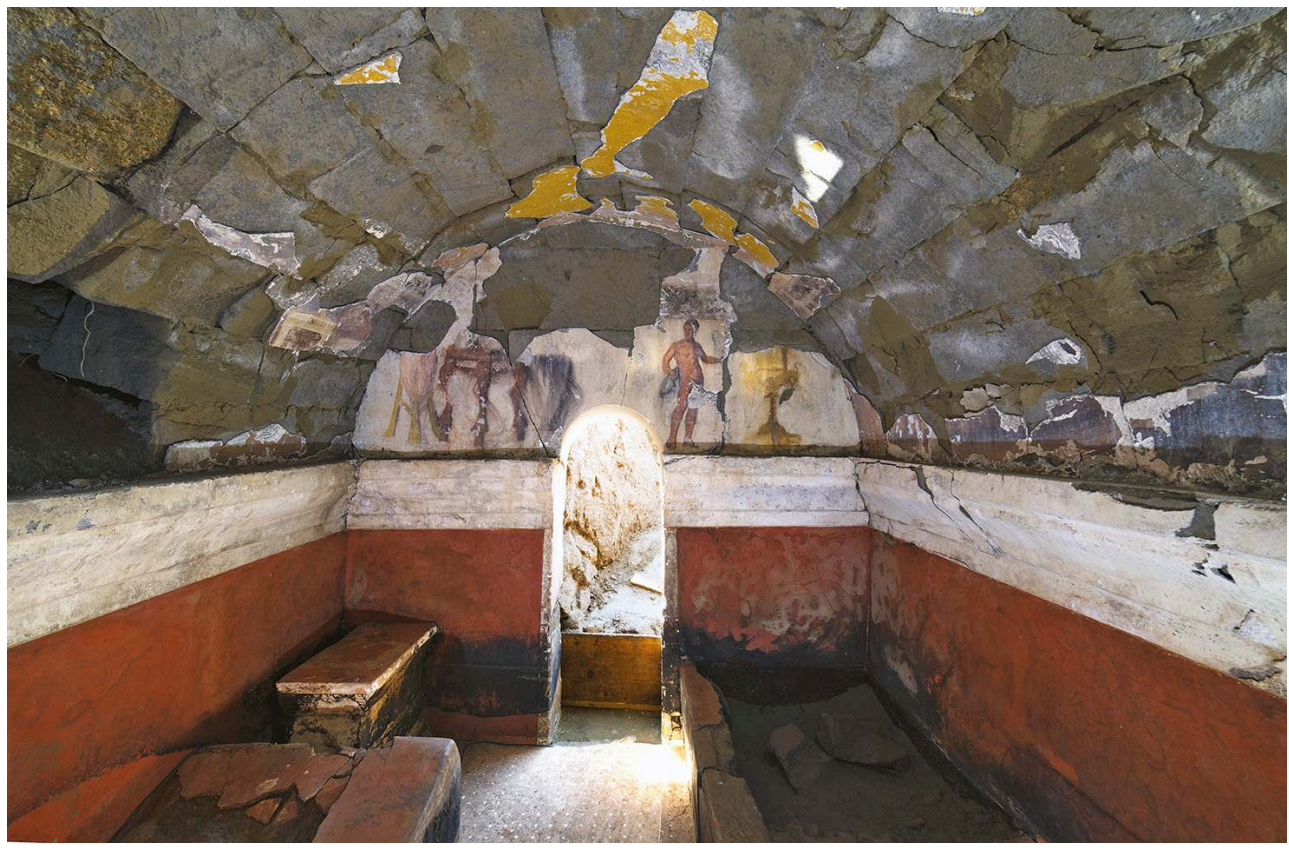

E. Lupoli, Archives CJB, CNRS-EFR / @ CC BY-NC-ND.

La pièce est organisée avec trois lits, disposés le long des murs ouest, nord et est (SP73129, SP73125, SP73127). La structure de chacun est constituée de la sorte : des grands blocs de tuf carrés sont posés de chant de manière à créer le périmètre, la zone est ensuite remplie d'un mélange de terre et de petits fragments de tuf jaune et enfin tous ces éléments sont recouverts d'un enduit rouge clair. Les lits sont également dotés d'un pulvinus à l'extrémité.

Dans le coin sud-est de la pièce, il y a une mensa rectangulaire. La structure est composée de deux blocs de tuf jaune superposés avec une moulure à cyma reversa à la base et au sommet, recouverte d'un enduit blanc sur les faces latérales visibles et d'un enduit rouge sur la face supérieure. Sur cette surface, sont visibles les traces en négatif des objets du mobilier funéraire qui y étaient placés.

Le seuil est constitué d'un bloc rectangulaire de tuf posé à plat et le pavement de la pièce est en mortier de tuileau peint et décoré de petites tesselles de calcaire blanc.

À l'intérieur, les murs de la chambre funéraire sont entièrement enduits et peints avec des couleurs unies ou des scènes figurées. La corniche moulurée est recouverte d'un enduit blanc, tandis que les murs en dessous sont de couleur ocre rouge, comme les lits mais dans une teinte plus claire. Une bande blanche encadre la porte d'entrée, dans la partie inférieure, se raccordant ainsi à la corniche.

41 Les reins de la voûte et les lunettes situées sur le mur d'entrée et sur le mur du fond, sont décorés d'une scène figurative et de représentations de paysages. La fresque n'a été conservée de manière exceptionnelle que dans la lunette sud-est, du côté de l'entrée, alors qu'elle est extrêmement fragmentaire et incomplète sur les trois autres murs.

42 Sur la lunette sud-est, une mégalographie représente une scène de préparation d'un banquet. À droite de la porte, un jeune serviteur, nu, tient dans sa main gauche un calice ou un kantharos à haut pied et dans la main droite une œnochoé, tous deux en 
argent. À gauche de la figure masculine, se dresse un cratère en calice sur un support, tous deux en métal doré.

À gauche de la porte, en revanche, on trouve une amphore de transport en céramique sur un trépied doré, une situle en argent et une table en bois, sur laquelle on devine au moins deux autres objets métalliques (?), probablement de la vaisselle dont peut-être un bol renversé. La taille du jeune homme est disproportionnée, beaucoup plus petite que les objets représentés à ses côtés.

Le mauvais état de conservation des enduits ne permet pas de décrire avec le même détail les représentations sur les murs latéraux et sur le mur du fond de la tombe de Cumes. Cependant, il semble y avoir une suite de paysages avec des édifices. La présence, dans les angles des parois, d'éléments architecturaux tels que des colonnes ou des piliers, représentés au premier plan par rapport au reste de la composition en arrière-plan, comme s'ils étaient posés sur la corniche moulurée, est évocatrice. Sur le mur est, sous une frise, on voit une partie d'un bâtiment, peut-être un naiskos, composé de deux colonnes avec des chapiteaux schématiques, une architrave à deux bandes et une corniche, au-dessus de laquelle semblent être représentés des acrotères ou des antéfixes ; au centre du fronton, un décor est peint d'un bleu intense.

Le dénominateur commun des différentes scènes sur l'ensemble des murs est la guirlande florale très dense, dans laquelle sont associés des feuilles, des fruits et quelques oiseaux.

L'intrados de la voûte, en revanche, est uniformément peint en jaune foncé et entouré d'une bande rose saumon, et semble rappeler une tenture ou la couverture d'un baldaquin.

L'étude du monument et de son décor en est encore à ses débuts, en attente surtout de la restauration de la lunette et de la recomposition du matériel fragmentaire provenant des autres parois. L'ensemble de l'appareil décoratif semble vouloir reproduire une scène de banquet en plein air dont les convives seraient les défunts étendus sur les lits funéraires. Le cadre, ainsi défini, pourrait être interprété comme un triclinium d'été entouré de colonnes, entre lesquelles il est possible d'observer des aspects du paysage environnant caractérisé par des éléments naturels et des édifices.

La fouille du monument a permis de récupérer les restes de mobiliers funéraires laissés, oubliés ou négligés par les fouilleurs du XIX ${ }^{\mathrm{e}}$ siècle (fig. 10). 
Fig. 10. Mobilier funéraire du tombeau à chambre hypogée MSL73101 .
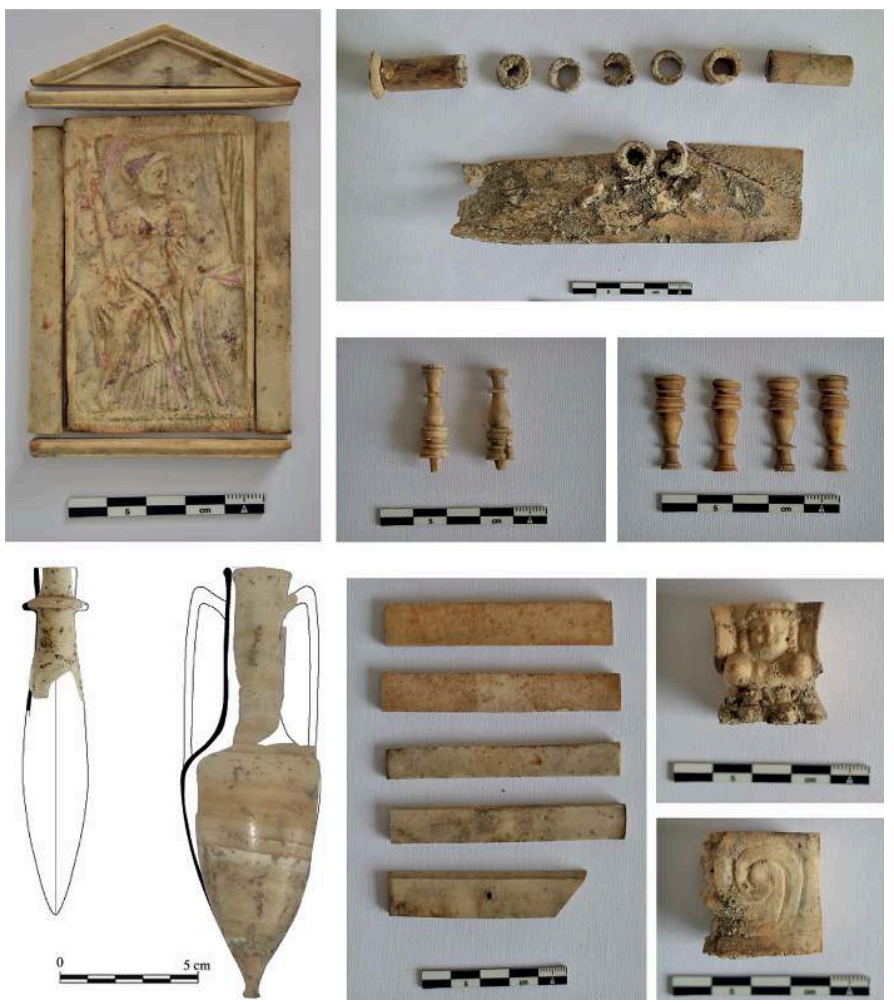

Archives CJB, CNRS-EFR / @ CC BY-NC-ND.
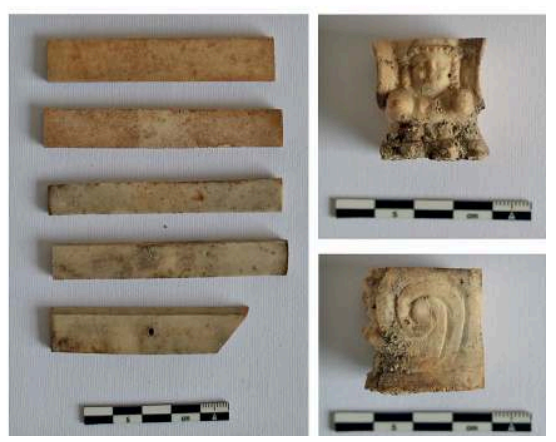

On y trouve notamment plusieurs fragments ainsi que quelques bandeaux fins d'os ouvragés qui pourraient faire partie du revêtement d'un coffret en bois. Une applique en os, de forme rectangulaire, finement travaillée, ornée d'une Minerve armée et assise, peut également être attribuée au même objet. La boîte devait être soutenue par des petits pieds en os sculptés en forme de patte griffue et ornés d'un sphinx dont un seul exemplaire a été retrouvé.

50 À l'intérieur de la chambre funéraire, plusieurs fragments appartenant à deux alabastra en albâtre ont également été trouvés, le premier de forme élancé, se terminant par une pointe et avec une lèvre droite moulurée (proche de la forme Colivicchi, classe II, type 1.3) datant de la seconde moitié du II et du début du Ir siècle av. J.-C. et le second, un amphorisque (attribuable à la forme Colivicchi, classe II, variante 2.2.2) datant de la première moitié du $\mathrm{I}^{\mathrm{er}}$ siècle av. J.-C. Il n'est pas exclu que le coffret puisse être interprété comme une alabastrothèque, c'est-à-dire un conteneur pour vases en albâtre, puisque les deux petits récipients que nous venons de décrire, dépourvus de base, avaient besoin d'un support pour tenir en position verticale.

Le mobilier funéraire devait être complété par d'autres objets dont seules des petites parties ont été retrouvées. Parmi ceux-ci, on trouve un fragment de miroir circulaire, plusieurs fragments de fer appartenant à un strigile et à un anneau porte-strigile, la partie finale d'un cure-oreille ou peut-être plus simplement d'une cuillère à cosmétique en métal (fer ?), l'extrémité moulurée d'un fuseau ou d'une épingle à cheveux en os, des fragments d'une quenouille en os sculptée, quelques maillons de chaîne en bronze, des objets probablement liés à des jeux, tels que deux dés en os et des pions en verre et en 
pierre, et enfin une lampe à huile à vernis noir, de forme biconique avec une anse (type Tevere $1 \mathrm{~b} / \mathrm{c}$ ).

Sur le plateau de la mensa, grâce à l'absence d'incrustations calcaires par endroits, on observe les négatifs des objets qui y étaient placés. Parmi ceux-ci, on peut reconnaître : un objet composé d'une poignée se terminant par une partie large et probablement plate (un flabellum? une épingle?); trois petits objets à base ronde, peut-être des alabastra à base plate ou des petites pyxides en os; dans le coin sud-est de la table, il reste des incrustations de bronze et les empreintes de ce qui aurait pu être une chaîne dans le même alliage; au milieu de la table, en revanche, il y a une trace rectangulaire qui pourrait appartenir à un coffret en bois (l'alabastrothèque ?) à laquelle certains des éléments en os mentionnés précédemment ont dû appartenir. À côté de ce coffret, on voit le négatif d'une forme quadrangulaire dont les dimensions correspondraient bien à celles d'une boîte pour miroir, en bois revêtu d'os, et dont on peut rapprocher de grands éléments plats, rectangulaires, en os.

53 L'ensemble de ce mobilier, trouvé à l'intérieur de la chambre funéraire, aide à supposer que le tombeau a été fréquenté durant la première moitié du $\mathrm{I}^{\mathrm{er}}$ siècle av. J.-C.

En fouillant le monument nous avons pu constater qu'il avait déjà été visité au moins deux fois au XIX siècle par des fouilleurs autorisés ou des clandestins. Deux trous d'accès sont visibles sur le mur du fond et sur le mur latéral est. À l'occasion de la première percée dans le monument, un des visiteurs a laissé sa signature et le dessin d'une œnochoé sur la lunette au décor figuré. Les recherches ont permis d'identifier le personnage et de dater l'une des deux interventions dans la période postérieure à l'unification de l'Italie. L'auteur de l'esquisse est Jacques-Alfred Bovet, commis de chancellerie au Consulat général de France à Naples, qui a mené des recherches dans la nécropole de Cumes en 1862-1863, avec le marquis de Gibot et le comte Tyszkiewicz ${ }^{16}$.

Les tombes à chambre avec voûte en berceau de la nécropole de la Porte médiane fournissent une série d'éléments - stratigraphie, technique de construction, matériaux, appareils décoratifs, données épigraphiques - qui permettent de dater le groupe d'hypogées cumains entre les dernières décennies $d u \mathrm{II}^{\mathrm{e}}$ et les premières décennies $d u$ $\mathrm{I}^{\mathrm{er}}$ siècle av. J.-C. Il s'agit d'une série de tombeaux qui se distingue des autres tombes à chambre à voûte en berceau de Campanie par ses caractéristiques, sa datation et son homogénéité ${ }^{17}$.

\section{Les tombes à crémation}

56 Les hypogées que nous venons de décrire coexistaient dans la nécropole de la Porte médiane avec des sépultures individuelles, de construction plus simple, qui témoignent d'un rite funéraire différent : l'incinération ${ }^{18}$. En retirant les niveaux de remplissage de la terrasse flavienne, dans la zone où se trouvent les deux hypogées MSL72006 et MSL73101, au moins sept marqueurs indiquant probablement des tombes à crémation en fosses avec déposition dans des réceptacles sont apparus (fig. 11). Il s'agit de blocs de tuf jaune parallélépipédiques, à base presque carrée ou rectangulaire, qui se répartissent sur toute la surface de la zone ouverte entre 2017 et 2020 et qui ne semblent pas suivre d'orientations prédéterminées ou former des alignements. Trois d'entre eux - SP73159, SP73162, SP73165 - sont flanqués d'un autre bloc de tuf, plus petit, disposé à l'horizontal, pouvant être interprété comme une table à offrandes (mensa). On note en particulier, la table de la sépulture SP73162, qui possède trois 
coupelles (diam. $8 \mathrm{~cm}$ ). Orientée ouest/sud-ouest/nord-est, la tombe est parmi les plus intéressantes, car elle porte, sur la partie supérieure de la pierre, un décor géométrique gravé que surmonte une inscription en osque, actuellement en cours d'étude. Deux autres tombes semblent avoir une orientation presque sud-nord: la tombe SP73159, près de la limite orientale de la zone de fouilles, juste au nord de l'axe routier V073161, présente une mensa sur le côté sud. Le cippe US 73159, qui a été rasé dans l'Antiquité, garde de faibles traces d'enduit blanc sur le côté ouest; sur le côté est, il est flanqué d'une amphore en position verticale, utilisée peut-être comme marqueur, ou comme une autre sépulture. Le bloc monolithe US 73164, près de la limite nord-ouest de la zone, présente un décor géométrique incisé sur la face sud, ce qui en détermine probablement son orientation. Le cippe US 73168, à l'ouest de MSL73101, montre une face sud encore partiellement couverte par une couche d'enduit. Au nord-ouest de la structure US 73168, se trouvent deux autres tombes : le cippe US 73165 avec une mensa, est positionné sud-est/nord-ouest; l'autre tombe (SP73167, constituée, semble-t-il, de deux blocs quadrangulaires superposés, s'inscrit presque entièrement dans le tracé de la voie en terre battue V073161, tandis que toutes les autres sont proches, mais légèrement détachées de l'axe. Dans aucun des cas, les niveaux de mise en place des sépultures n'ont été atteints, c'est pourquoi une datation plus précise et une meilleure compréhension des relations stratigraphiques ne seront possibles qu'avec la poursuite des fouilles.

Fig. 11. Photogrammétrie de la zone E73.

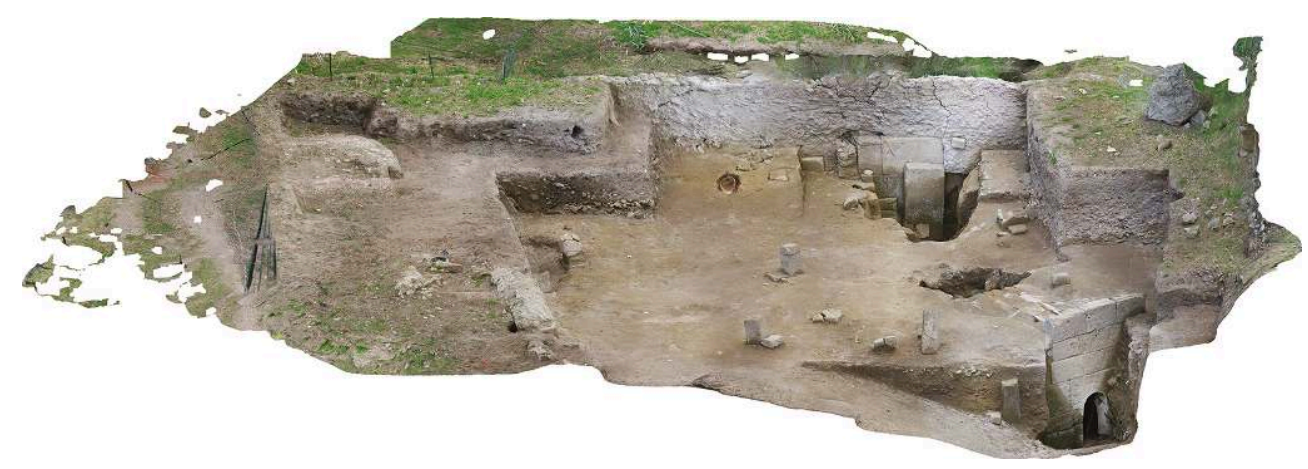

G. Sachau-Carcel, Univ. Paul-Valéry Montpellier 3, UMR 5140 ASM /

(c) CC BY-NC-ND.

\section{La nécropole entre le deuxième quart du I $^{\text {er }}$ siècle av. J.-C. et l'époque augustéenne}

Durant la première moitié du I ${ }^{\mathrm{er}}$ siècle av. J.-C., les tombes à voûte en berceau faites de gros blocs de tuf sont remplacées par des monuments semi-hypogées édifiés en opus incertum et recouverts d'enduit, destinés à accueillir des inhumations (fig. 12). La partie visible est comprise entre 0,60 et $0,80 \mathrm{~cm}$ d'élévation. Il y a plusieurs aspects techniques et constructifs que les nouvelles tombes ont en commun avec celles que nous venons de décrire. Et à cet égard, il est utile de rappeler la deuxième phase de construction de la tombe MSL72006 au cours de laquelle la voûte en blocs de tuf est stabilisée avec la réalisation d'un toit à double pente recouvert sur le dessus par du mortier de tuileau ; il 
s'appuie sur deux petits murs latéraux qui ont un parement extérieur en opus quasi reticulatum ${ }^{19}$.

Cette typologie de monuments est déjà documentée dans la nécropole de la Porte médiane et, à ce titre, citons les mausolées MSL29027 et MSL33001²0, situés un peu plus au nord le long de l'axe nord-sud (D), à proximité immédiate de la tombe à voûte en berceau MSL25027, fouillée par Andrea de Jorio en 1818 et redécouverte en $2010^{21}$.

Fig. 12. Les mausolées MSL46501, MSL46420, MSL46522 et MSL73178.

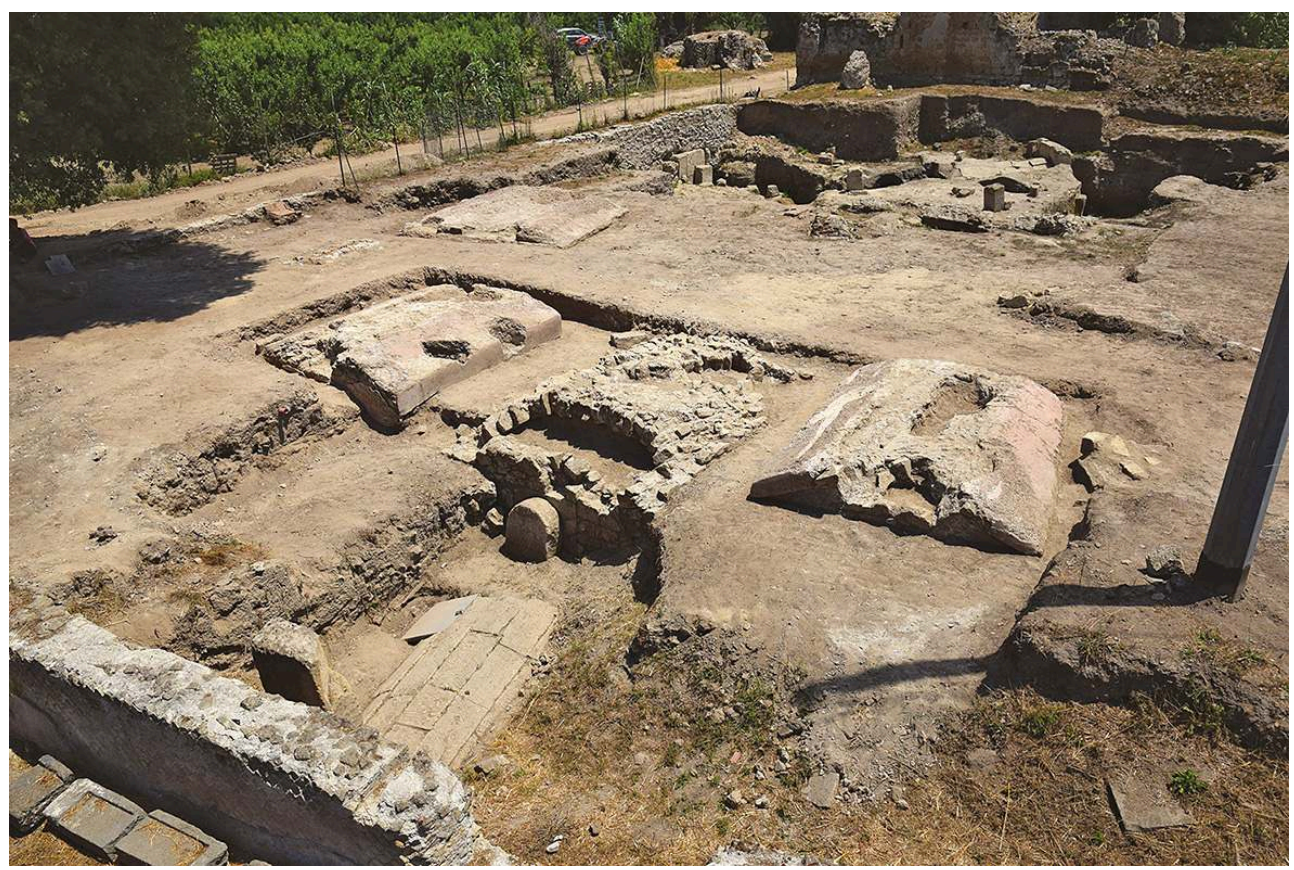

Archives CJB, CNRS-EFR / C CC BY-NC-ND

Le monument funéraire à chambre semi-hypogée MSL60321, exploré en 2019, rappelle d'un point de vue typologique le tombeau MSL29027 (fig. 13-14)22. Il n'a pu être fouillé que sur son tiers oriental. Orienté est-ouest, le monument mesure $2,30 \mathrm{~m}$ de large et ouvre vers le nord sur un passage qui n'a pas encore pu être mis au jour. Il est construit en opus incertum avec des pierres de tuf de forme irrégulière, de petites et moyennes dimensions, liées au mortier et disposées sans assises. La couverture de la voûte, qui devait avoir un revêtement en mortier de tuileau, est très peu conservée. L'accès à la chambre, enduite de blanc, se faisait par une ouverture placée dans la partie orientale du mur nord, dotée de deux piédroits et d'un linteau monobloc cintré, fermée au moment de la découverte par un monolithe de tuf (fig. 15). L'intérieur, de plan rectangulaire et d'orientation est-ouest, avec un sol en mortier de tuileau, était organisé avec au moins un lit disposé le long du mur sud, constitué d'un muret estouest, construit en pierres de tuf liées au mortier, ainsi qu'avec une structure bâtie remplissant l'espace du lit funéraire et servant à recevoir le défunt. L'ensemble, dégagé sur $0,60 \mathrm{~m}$ de longueur, est recouvert d'un enduit rosé et apparaît plus élevé vers l'ouest, du côté de la tête du défunt. Le long des murs, une corniche en saillie à profil simple sur laquelle repose la voûte en berceau est constituée de moellons de tuf réguliers, rectangulaires, disposés de chant et selon un schéma radial. À l'extérieur, 
au-dessus de l'entrée, un cippe englobé dans la maçonnerie est visible. De ce côté, la mise en œuvre présente une plus grande régularité dans les dimensions du matériau constitutif et en correspondance de l'entrée, à cause des piédroits et du linteau de la porte, les moellons sont disposés en assises.

Fig. 13. Le mausolée MSL29027.

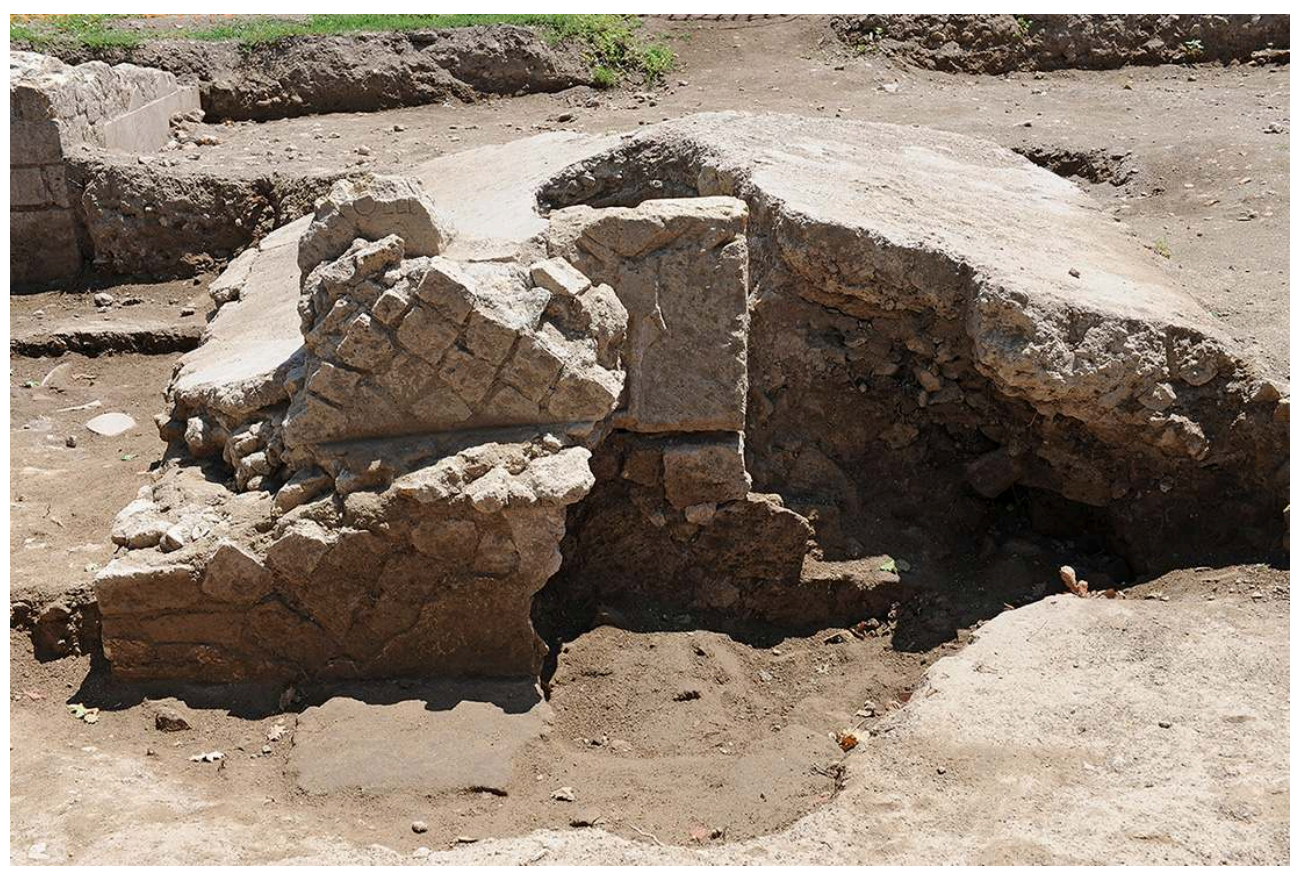

Archives CJB, CNRS-EFR / C CC BY-NC-ND

Fig. 14. L'intérieur du monument funéraire MSL29027.

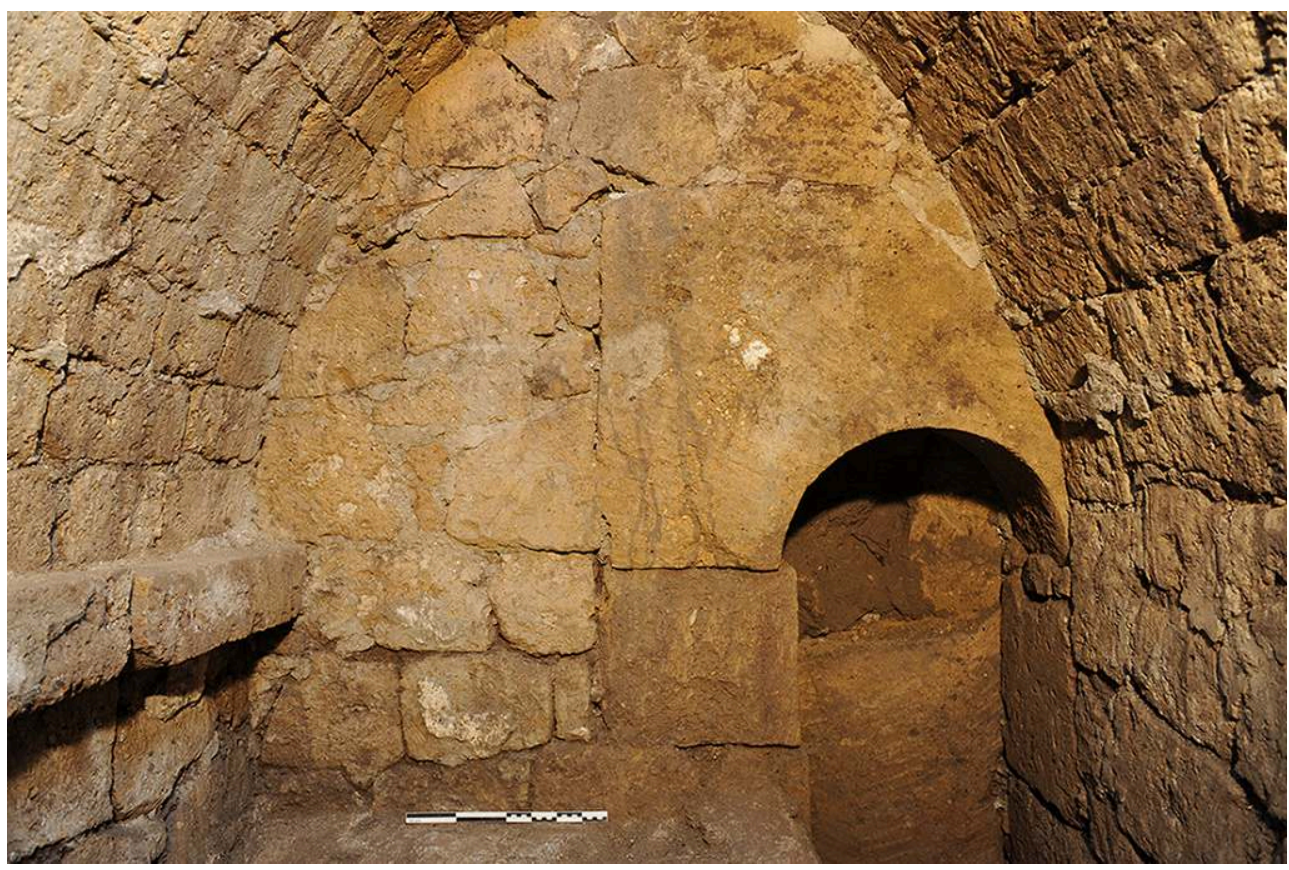

Archives CJB, CNRS-EFR / @ CC BY-NC-ND 
Fig. 15. L'intérieur du monument funéraire MSL60321.

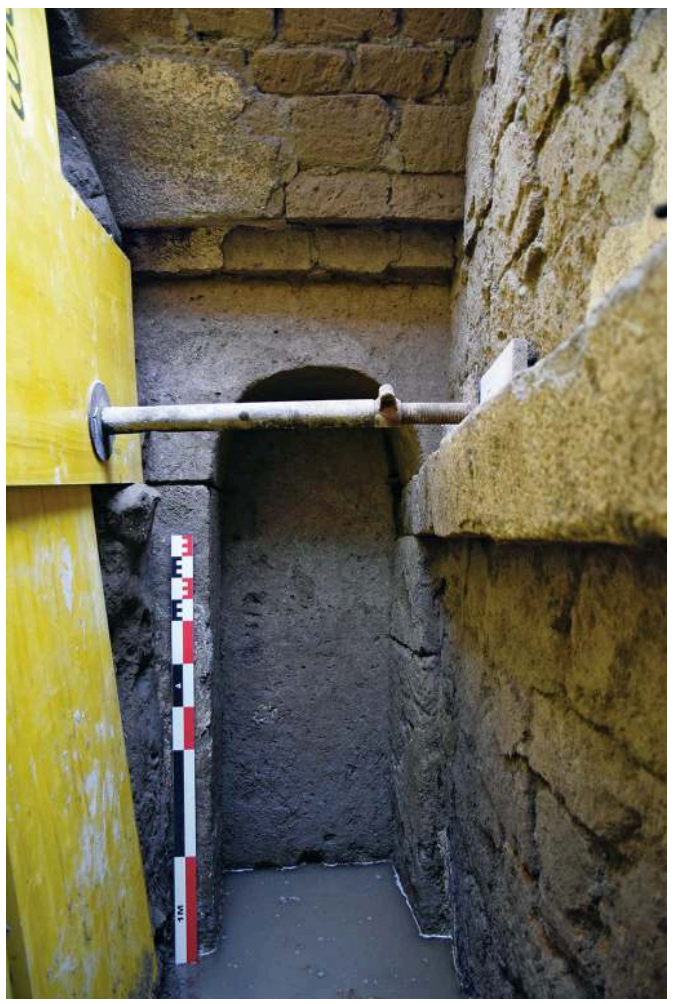

Archives CJB, CNRS-EFR / @ CC BY-NC-ND.

60 À l'intérieur du monument, bien qu'il ait déjà été pillé, la fouille a permis de récupérer quelques objets du mobilier funéraire, parmi lesquels des alabastra en albâtre, une coupe à paroi fine (forme Mayet X / Marabini XXV), des unguentaria en céramique (types CUM-UNG A et CUM-UNG B.IVB), des pions de jeu en pâte de verre, un dé en os, des fragments d'un strigile en fer, des anneaux en bronze et des éléments métalliques appartenant vraisemblablement à des coffrets en bois. Les objets permettent de placer la construction et une première fréquentation du tombeau entre le deuxième quart et le troisième quart du I ${ }^{\text {er }}$ siècle av. J.-C. La découverte d'un balsamaire en verre (De Tommaso 1990, type 19 = Ising 28a) atteste l'utilisation de ce monument encore durant le premier quart du $\mathrm{I}^{\mathrm{er}}$ siècle apr. J.-C.

61 Une série de monuments funéraires identifiés au cours des campagnes de fouilles de 2019-2020, entre le secteur occupé par les deux tombes à voûte en berceau MSL730101 et MSL72006 et la zone située devant la Porte, peut être rattachée à la même phase. Il s'agit des mausolées MSL46501, MSL46420, MSL46522 et MSL73178. Semi-enterrés, ils sont disposés sur deux rangées (fig. 12).

62 La première rangée est située immédiatement à l'est de la tombe à voûte en berceau MSL46070, fouillé lors de la campagne de $2012^{23}$. Les monuments sont orientés avec leurs façades vers la voie nord-sud (D) qui sort de la Porte médiane.

- Le premier de la série est le mausolée MSL46501, de plan quadrangulaire (dimensions extérieures $3,40 \times 3,50 \mathrm{~m}$ ) et orienté d'ouest en est. Les murs de l'édifice sont en opus 
incertum avec des moellons de tuf, de différentes dimensions et de forme irrégulière, liés avec du mortier et recouverts d'un enduit rose. L'accès au monument devait se faire par l'ouest, par une porte située au milieu de la façade. Il conserve un toit à double pente recouvert d'une épaisse couche de mortier de tuileau, revêtu d'un enduit peint en rouge (deux couches distinctes sont visibles). Le monument a subi de nombreuses spoliations et a été visité à plusieurs reprises par des fouilleurs clandestins, comme en témoignent les différents creusements présents en correspondance de la voûte. Sur le haut de la façade, le négatif d'un bloc est visible, très probablement un cippe ou une stèle. L'intérieur n'a pas été exploré.

- Le deuxième monument, MSL46420, est situé immédiatement au sud du précédent, derrière la tombe à voûte en berceau MSL46070; il est plus endommagé que les autres ${ }^{24}$. Orienté ouest-est et de plan quadrangulaire (dimensions extérieures $3,25 \times 4,10 \mathrm{~m}$ ), il est en opus incertum constitué de moellons et blocs de tuf de forme polygonale irrégulière, de tailles différentes, assemblés de manière désordonnée et liés au mortier. La voûte, dont la partie occidentale manque, est constituée de moellons de tuf rectangulaires, disposés de chant selon un schéma radial. L'intérieur de la chambre funéraire est recouvert d'un enduit fin blanc, lissé. Le monument a un accès du côté ouest, matérialisé par une porte qui présente un linteau monobloc et des traces d'enduit sur le parement externe. L'entrée est fermée par un bloc de tuf. L'organisation interne du monument n'a pas pu être observée.

- Le troisième, le monument MSL46522, est le dernier de la rangée et il est orienté vers le sud ; il a été pillé à plusieurs reprises (deux grandes ouvertures sont visibles sur le toit et sur le haut de la façade). Il est orienté ouest-est et semble mieux conservée que les autres. De plan quadrangulaire (dimensions extérieures 3,50 x 2,75-2,95 m), il est construit en moellons de tuf de forme irrégulière, liés au mortier ; le tout est recouvert d'un enduit blanc. Il a un toit à double pente recouvert de mortier de tuileau peint en rouge. Sur le haut de la façade, à l'ouest, vers la voie nord-sud (D), est visible l'empreinte en négatif d'un cippe ou d'une stèle. De ce côté, le monument possède une corniche en saillie de profil rectangulaire qui suit la double pente du toit. La voûte, visible sur le côté est, est constituée de moellons de tuf réguliers, rectangulaires, posés de chant et disposés selon un schéma radial. L'intérieur n'a pas été exploré.

63 La deuxième rangée, immédiatement à l'est, est composée, en l'état actuel des recherches, de deux monuments.

- Le mausolée MSL73178 est situé derrière la tombe MSL46501 et semble appartenir à la même typologie. Il est un peu plus grand que les autres et a un plan carré (4 m par côté). Orienté ouest-est, son accès devait se faire par la façade ouest. Le mur de façade, dont seule la partie supérieure a été mise en lumière, est constitué de gros blocs de tuf et, en son centre, un négatif indique la présence, à l'origine, d'un cippe ou d'une stèle. Le monument semble avoir connu au moins deux phases de construction. La seconde correspond à une rénovation des murs périmétraux avec un doublement des parois sur les côtés nord, est et sud en opus incertum/opus quasi reticulatum avec des moellons et des cubilia, avec un alignement irrégulier. Le toit est à double pente et recouvert d'une épaisse couche de mortier qui recouvre les doublements de murs dont la surface est peinte en rouge. Le monument a subi de nombreuses spoliations qui sont visibles en plusieurs endroits.

- La dernière tombe, le monument MSL73184, est la plus endommagée, car elle est en grande partie rasée, donc, sans partie supérieure. Elle est située immédiatement à l'est du tombeau MSL46420. Son orientation, sud-nord, diverge de celle des autres tombeaux et l'accès devait se faire par le sud. De plan quadrangulaire, elle est construite en opus incertum avec des pierres de tuf de petit module, de dimensions inégales, de forme irrégulière, assemblées avec 
du mortier et disposées sans assises, avec un chaînage d'angle en moellons de tuf rectangulaires. Les murs sont recouverts d'un enduit blanc, visible sur la paroi orientale, la seule bien conservée. L'intérieur de la chambre funéraire est recouvert d'une fine couche d'enduit blanc, lissée. Ce monument est probablement le plus récent de la série.

- Pour une meilleure compréhension de cette typologie de monuments funéraires, il convient de mentionner le mausolée MSL33001, situé environ 50 mètres plus au nord, le long de l'axe nord-sud (D), et qui a fait l'objet d'une fouille en 2010 (fig. 16-17) ${ }^{25}$. Déjà altéré au moment de la découverte, il était cependant encore bien conservé. Orienté ouest-est, il a un plan presque quadrangulaire (extérieur 3,00 x 2,70 m ; intérieur 2,65 x 2,35 m). À l'extérieur, la partie exposée (environ $0,85 \mathrm{~m}$ ) est recouverte d'une couche d'enduit blanc. La voûte est enduite d'une épaisse couche de mortier de tuileau qui s'appuie sur une corniche en saillie composée d'une rangée de moellons de tuf de forme rectangulaire. Dans le toit, un grand trou a été fait par les fouilleurs clandestins. La chambre est couverte d'une voûte en berceau, faite de pierres de tuf de petit module et dimensions régulières, liées au mortier et disposées selon un schéma radial ; elle repose sur une corniche en saillie. Trois lits funéraires sont construits le long des murs nord, est et sud; ils sont formés par des murets en pierres de petits module, assemblées au mortier et à la terre, et par un remplissage de terre, et revêtus d'un enduit rouge. Les parois portent un enduit blanc et le sol est en mortier de tuileau. Aucun élément du mobilier funéraire n'a été retrouvé, mais la séquence stratigraphique confirme une fréquentation du monument entre le deuxième quart et le troisième quart du $\mathrm{I}^{\mathrm{er}}$ siècle av. J.-C.

Fig. 16. Les mausolées MSL25027 et MSL33001.

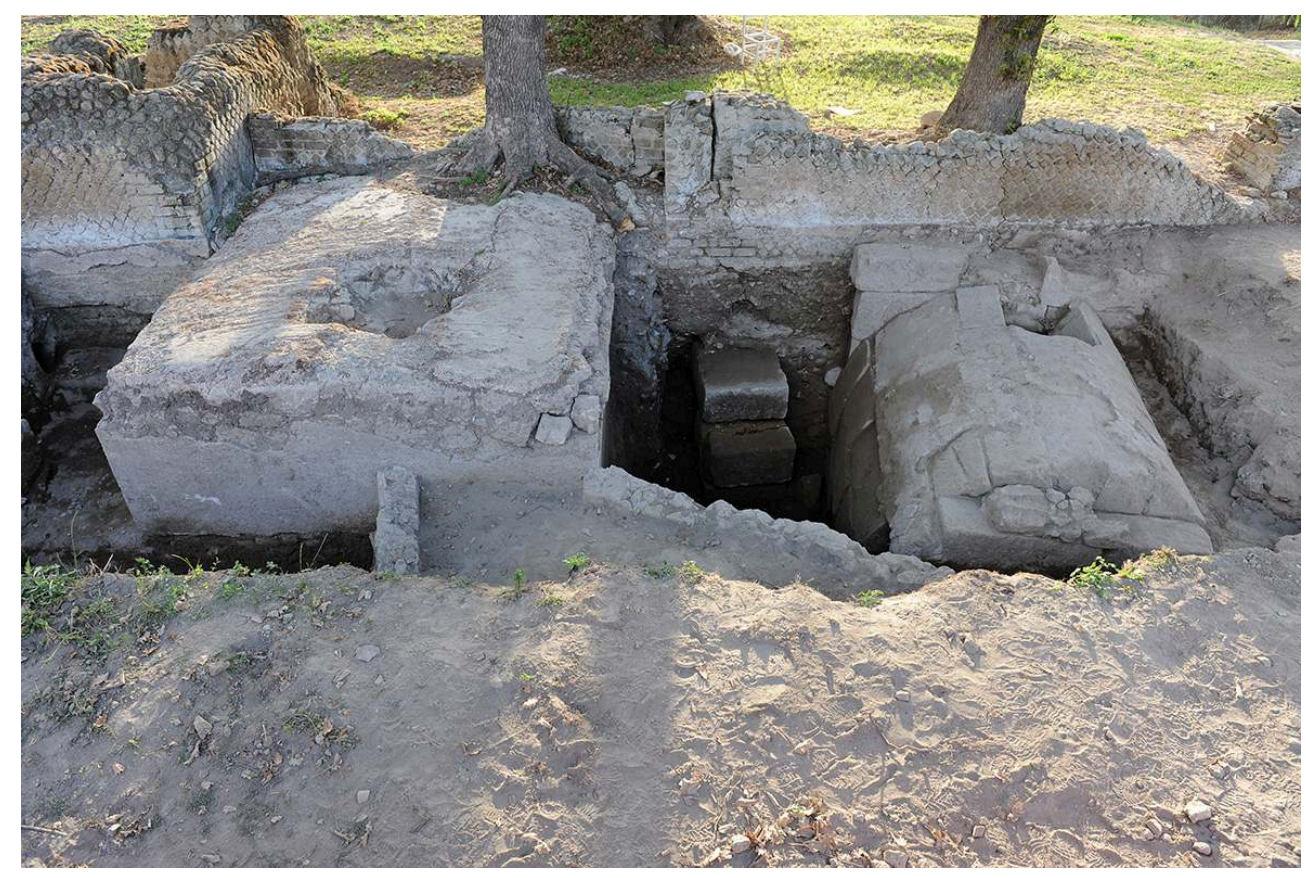

Archives CJB, CNRS-EFR / @ CC BY-NC-ND. 
Fig. 17. L'intérieur du monument funéraire MSL33001.

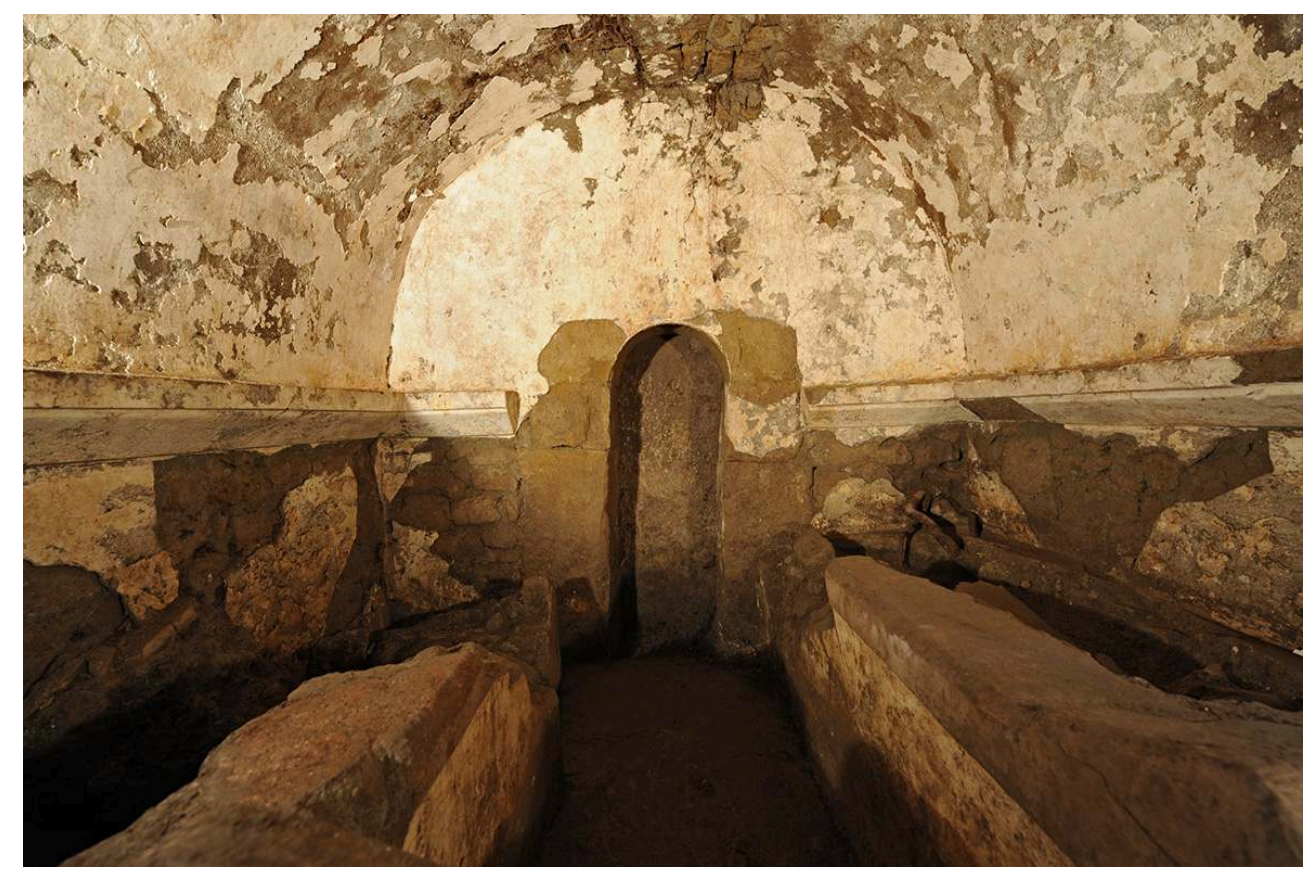

Archives CJB, CNRS-EFR / @ CC BY-NC-ND.

64 L'absence de structures funéraires au-delà de l'alignement composé des monuments funéraires MSL46522, MSL73184 et MSL73101, ainsi que la présence d'une succession de niveaux de sols devant ces derniers, semblent indiquer la présence d'une voie ouest-est et l'existence d'une zone non construite devant les murs.

Un peu plus tard et dans la seconde moitié du I ${ }^{\text {er }}$ siècle av. J.-C., dans le secteur proche de la Porte, le long de l'axe nord-sud (D), est construite une autre série de monuments. Il s'agit des mausolées MSL46346, MSL46144, MSL46175, MSL60111 et MSL60137. Les trois premiers ont été étudiés lors des campagnes 2012 et 2013.

- Au premier rang, le plus proche de l'axe routier nord-sud, se trouve uniquement le monument MSL46346, étudié en 2013 (fig. 18a) ${ }^{26}$. Le bâtiment n'est que partiellement préservé, car il a été détruit par la construction du mur de soutènement de l'espace dallé devant la Porte. La face intérieure des murs est en opus incertum/quasi reticulatum avec pierres de petit module et des cubilia de tuf de grandes dimensions, à parois pleines, sans chaînage d'angle, tandis que l'extérieur est construit contre terre ; le monument était donc en partie enterré.

Les sépultures MSL46144 (fig. 18b) et MSL46175 (fig. 18c), toutes deux étudiées en 2012, appartiennent à une deuxième ou troisième rangée ${ }^{27}$.

- Le mausolée MSL46175, le plus ancien des deux, n'a été que partiellement exploré car il a été rasé et recouvert par les pièces du complexe impérial. Le mur de façade, à l'ouest, est en opus incertum de pierres de petit module et de forme irrégulière. L'accès se faisait par une porte, encore fermée par un monolithe de tuf, dont les piédroits en blocs de tuf ont été spoliés. La largeur extérieure du monument est de $3,20 \mathrm{~m}$, tandis que la largeur intérieure est de 2,50 $\mathrm{m}$. La chambre était recouverte d'une couche d'enduit blanc. 
Fig. 18. Les mausolées MSL46346 (a), MSL46144 (b), MSL46175 (c).
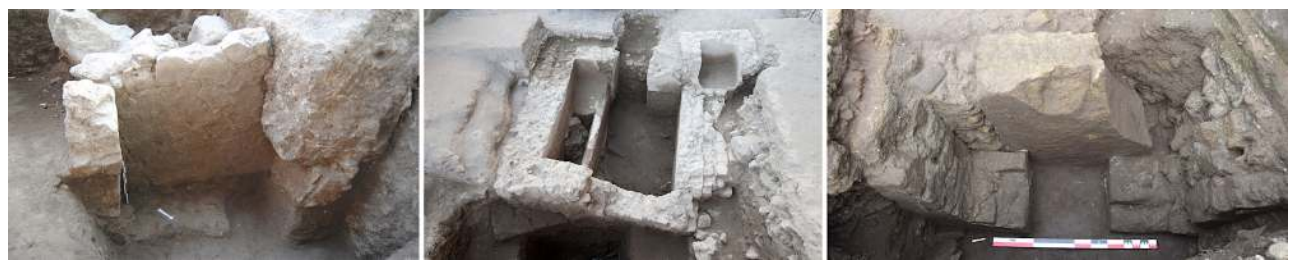

Archives CJB, CNRS-EFR / @ CC BY-NC-ND

- Le mausolée MSL46144, qui est également semi-hypogée, a été installé contre le mausolée MSL46175. De forme légèrement trapézoïdale ( 3 × 2,75 x 3 x 2,95 m), il est construit contre terre, ne présente pas de parement extérieur et, à l'intérieur, il est en opus quasi reticulatum fait avec des cubilia de grandes dimensions, de forme pas toujours régulière ; les murs n'ont pas de chaînage d'angle et seul l'intérieur présente un revêtement d'enduit. La surface de la chambre est de $3,9 \mathrm{~m}^{2}(2 \times 2 \times 2,06 \times 1,92 \mathrm{~m})$. L'accès se faisait par l'ouest à travers un dromos d'environ $1 \mathrm{~m}$ de long et $1,40 \mathrm{~m}$ de large. La fouille a permis d'identifier au moins deux phases de construction qui correspondent probablement à deux phases d'utilisation du monument funéraire. Durant la première phase, on note une structure semi-circulaire construite en mortier et adossée au centre du mur sud qui conserve un négatif de forme circulaire dans la partie supérieure. Certaines traces suggèrent la présence de structures similaires également au milieu des murs nord et est (supports pour les urnes cinéraires?). Dans la seconde phase, la chambre funéraire subit des transformations et les structures semi-circulaires sont oblitérées par la construction de deux lits funéraires le long des murs sud et nord ; parmi ceux-ci, seul le lit sud (SP46150) a été conservé, tandis que le lit nord se devine en négatif. On peut attribuer à cette même phase la construction d'un sol de mortier de tuileau, qui a été complètement détruit.

Les derniers de la série sont les mausolées MSL60137 et MSL60111, situés au-delà des limites de l'espace dallé ménagé devant la Porte médiane. Leur construction remonte à la première période augustéenne.

- Le monument funéraire MSL60137, fouillé lors des campagnes de 2017 et 2018, est à chambre semi-hypogée, d'un plan presque carré (fig. 19) ${ }^{28}$. Il est couvert d'une voûte en berceau et il était accessible de l'ouest par une porte qui est encore aujourd'hui obstruée par un grand bloc de tuf. Les murs sont bâtis avec un parement en opus quasi reticulatum qui conserve par endroits un revêtement de mortier de chaux et sable avec des traces d'un décor peint à motifs végétaux (?) ; La chambre funéraire a un sol en béton de tuileau dont la surface est peinte en rouge. Elle comporte trois lits maçonnés, disposés en U le long des murs. Ils sont délimités par des murets revêtus d'un enduit clair définissant un espace rempli de terre. Le monument a été perturbé par des pilleurs. L'ensemble des objets découverts sur les lits confirme une utilisation du monument durant le premier quart du $\mathrm{I}^{\mathrm{er}}$ siècle apr. J.-C. 
Fig. 19. Vue de l'intérieur du mausolée MSL60137.

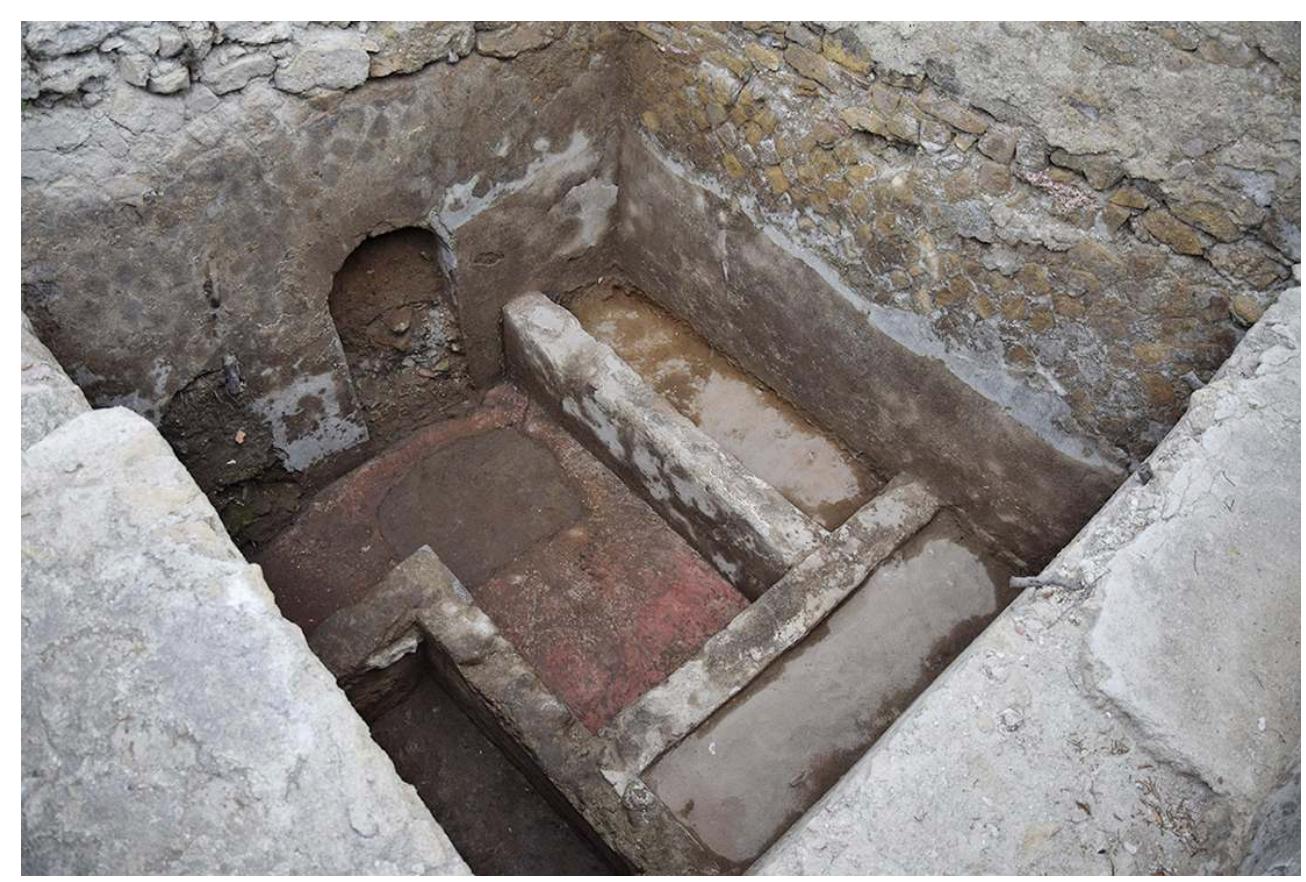

Archives CJB, CNRS-EFR / @ CC BY-NC-ND.

- Le monument funéraire MSL60111 a été repéré en plan en $2016^{29}$ et fouillé en $2018^{30}$, hormis la partie se trouvant sous l'emprise des structures du comptoir de la boutique du complexe trajanien ENS46104 (fig. 20). Le niveau d'arase du bâtiment, à hauteur des lits funéraires, a laissé subsister les murs : à l'intérieur les murs sont bâtis avec un parement en opus quasi reticulatum; ils présentent des traces de banchages à l'extérieur ; le monument était donc en partie enterré. Il abritait une chambre funéraire à laquelle on accédait depuis le sud par une porte. Ce mausolée présente un plan classique avec trois lits funéraires maçonnés, construits contre les murs est, nord, et ouest, sur un sol de béton gris lissé et laissant un espace libre au centre, ainsi que dans l'angle sud-est de la pièce. L'ensemble des murs de la pièce et des lits funéraires est recouvert d'un enduit. Les nombreux objets de mobilier découverts sur les lits et dans l'espace laissé libre au sud-est de la chambre funéraire confirment la chronologie et attestent une utilisation du tombeau jusqu'au milieu du I ${ }^{\text {er }}$ siècle apr. J.-C. 
Fig. 20. Vue zénithale du mausolée MSL60111.

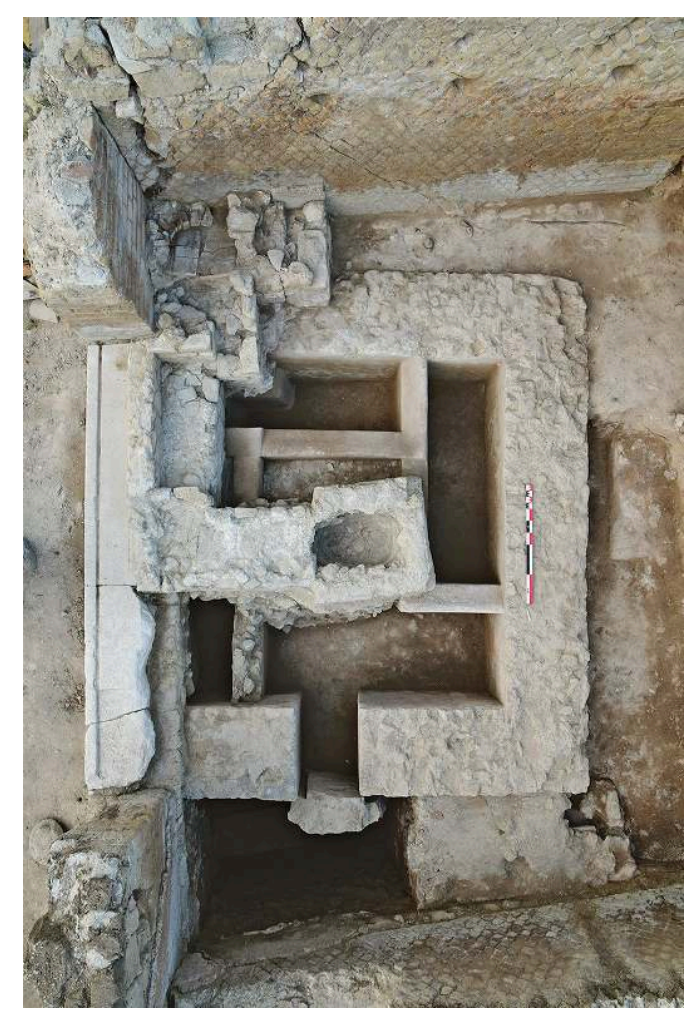

Archives CJB, CNRS-EFR / @ CC BY-NC-ND.

\section{Les remparts (fig. 21-22)}

Au cours des campagnes 2018 et 2019, une série de sondages stratigraphiques a été réalisée en limite méridionale de la terrasse (Zone E74), afin de mieux comprendre l'évolution topographique de la nécropole par rapport aux fortifications et au fossé défensif ${ }^{31}$. Les dégagements ont permis de mettre au jour le renforcement de l'enceinte hellénistique (RMP74082, RMP74013) et un tronçon du mur d'époque tardo-archaïque (RMP74097). Malheureusement, dans l'Antiquité tardive et à l'époque moderne, au début des années 1890, à l'occasion de la construction du grand réseau d'égouts de Naples, les murs ont subi une spoliation considérable dans ce secteur ${ }^{32}$.

Le doublement hellénistique (RMP74082) a été intercepté sur une longueur d'environ $23 \mathrm{~m}$, mais seulement à l'extrémité est, où il est conservé sur une plus grande hauteur. L'élévation composée de huit assises a été partiellement dégagée, alors que les fondations n'ont pas encore été atteintes. Le mur se compose d'une courtine externe faite de grands blocs de tuf jaune à assise plate isodome, a secco, sans liant et présentant un profil externe en gradins; la partie postérieure, se développe avec des murets transversaux longs de 2,65 m et distants de 1,65 m à 2,05 m, remplis d'un emplekton fait d'éclats de taille tuf jaune de grandes dimensions, disposés dans une matrice composée de limon et de sable. Les blocs ont une longueur variable entre 0,58 et $1,70 \mathrm{~m}$, une largeur entre 0,73 et $0,82 \mathrm{~m}$ et une épaisseur entre 0,38 et $0,41 \mathrm{~m}$. La surface de la tête des blocs montre encore les traces qui ont été faites par les leviers lors de l'installation des blocs de pierre de l'assise supérieure. La datation de cette phase fixée à la première moitié du III ${ }^{e}$ siècle av.J.-C., déjà avancée par les fouilles de l'équipe de l'Université 
L'Orientale de Naples, est confirmée par les fragments de céramique retrouvés dans les couches fouillées de l'emplekton.

Fig. 21. Vue zénithale du secteur des remparts.

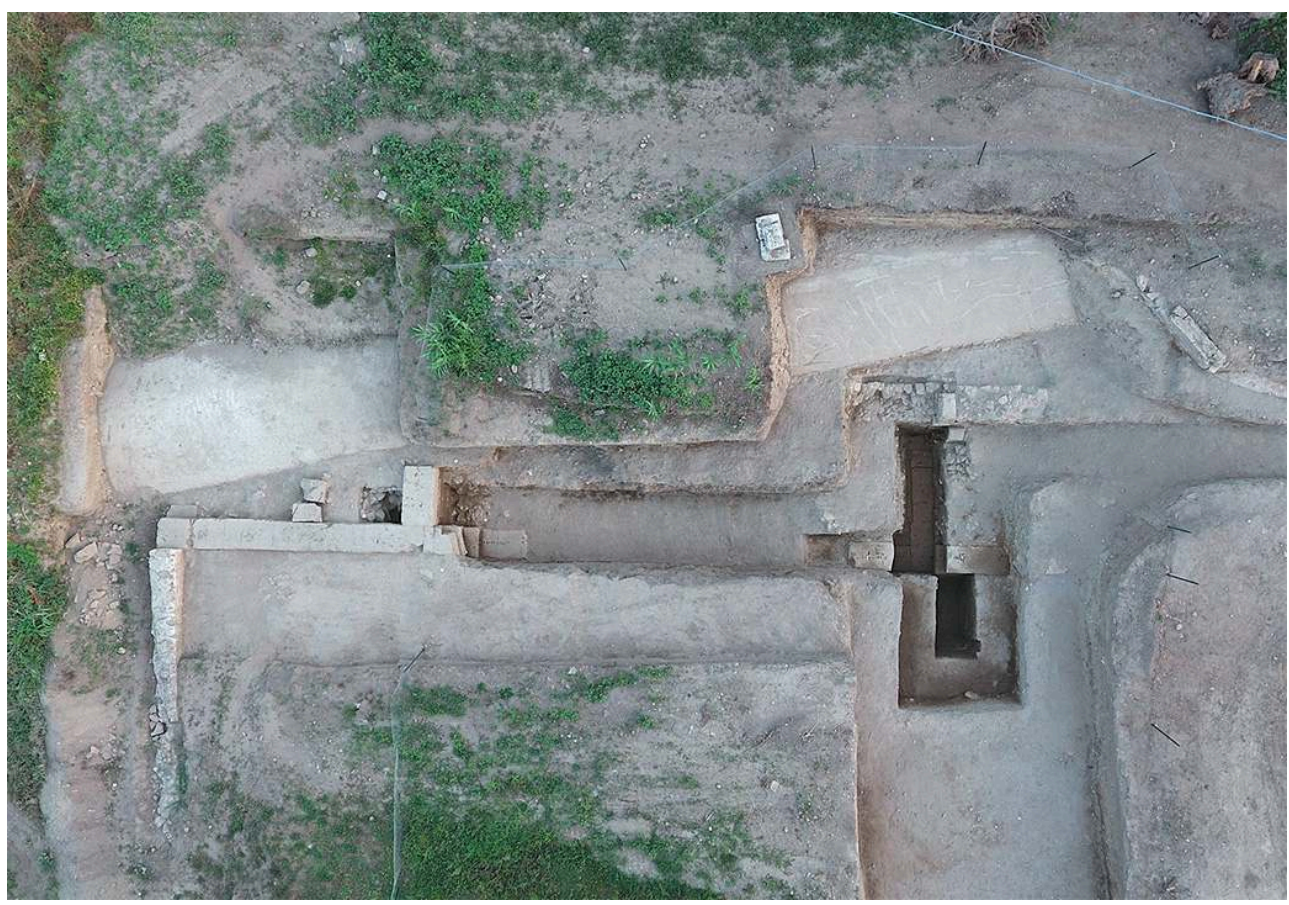

E. Botte, CNRS, CCJ / @ CC BY-NC-ND. 
Fig. 22. Les fortifications (Zone E74).

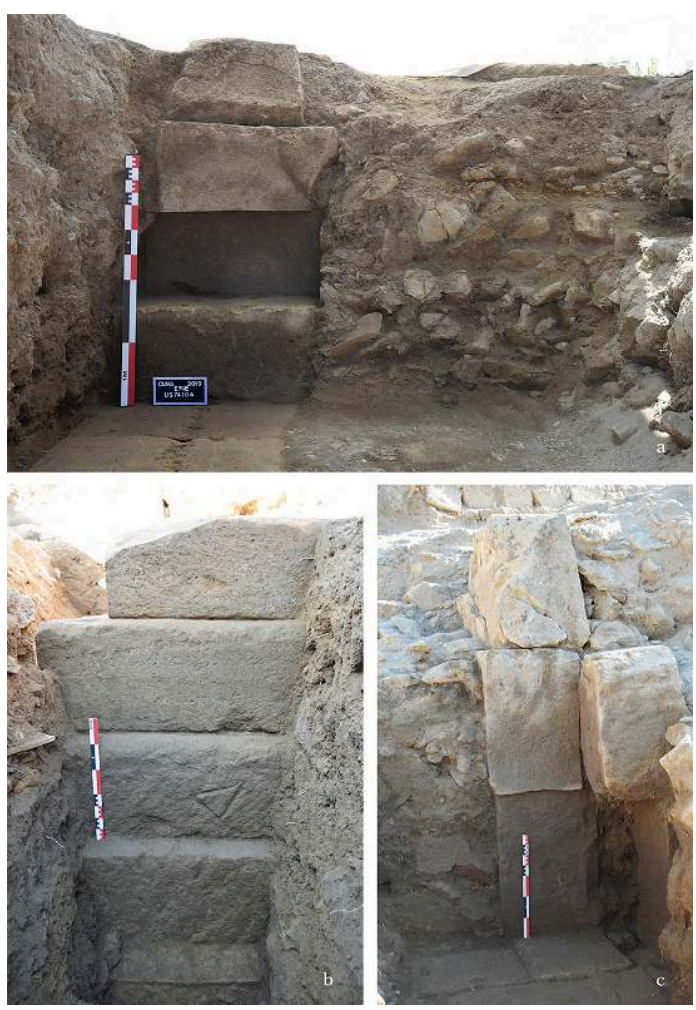

a. Le rempart hellénistique RMP74013 et l'emplekton US 74104, vu de l'est ; b. Vue frontale du parement extérieur du rempart hellénistique (RMP74082) ; c. Le rempart tardo-archaïque (RMP74097), vu de l'ouest.

Archives CJB, CNRS-EFR / (c) CC BY-NC-ND.

69 L'enceinte hellénistique s'appuie directement sur le mur tardo-archaïque (RMP74097). Dans le sondage ouvert à l'ouest, ce dernier n'a conservé que la courtine extérieure, formée de grands blocs de tuf jaune, réalisés en orthostates avec un profil en pente. Celle-ci présente des murets transversaux longs d'environ $0,90 \mathrm{~m}$, entre lesquels se développe une structure pleine, en mortier de terre et éclats de tuf jaune.

La fouille s'est également concentrée sur un sondage réalisé devant la courtine de l'enceinte hellénistique (RMP74082) qui a permis d'identifier quelques niveaux de remblais d'époque flavienne. Ceux-ci viennent combler un premier niveau de circulation d'époque tardo-républicaine (SL74093). Sous ce niveau, un sol contemporain de la muraille hellénistique a été identifié, qui vient lui-même recouvrir un niveau composé d'éclats de tuf jaune, témoignant de la présence d'un niveau de chantier à mettre en relation avec la taille des blocs utilisés pour la construction de la courtine.

\section{Conclusion}

71 La zone de la Porte médiane des fortifications septentrionales a subi un réaménagement majeur entre la fin du $\mathrm{I}^{\mathrm{er}}$ et le début du $\mathrm{II}^{\mathrm{e}}$ siècle apr. J.-C., accompagné de la construction de la via Domitiana et de la monumentalisation de la cour devant celle-ci ${ }^{33}$. Au cours de ces grands travaux, le secteur situé immédiatement à l'est de la Porte est rehaussé pour la réalisation d'une grande terrasse. Ces opérations avaient pour but d'aplanir une grande partie du terrain, utile pour pouvoir construire un 
complexe doté d'un espace extérieur considérable, interprété comme un campus. Cet ensemble couvre au moins $2500 \mathrm{~m}^{2}$ et possède encore, au nord et à l'est, les murs de soutènement conservés en fondations, tandis qu'au sud, il s'appuie directement sur le doublement des fortifications d'époque hellénistique. Au cours de cette phase, l'axe routier $\mathrm{E}$ en direction du Monte Grillo est occulté et le secteur des nécropoles à l'est est englobé dans le remblai de la terrasse.

Durant les premières décennies du $\mathrm{II}^{\mathrm{e}}$ siècle apr.J.-C., le complexe est doté d'un portique et d'une série de pièces, dont certaines à but commercial (la boutique BTQ60066), donnant sur l'espace dallé ménagé devant la Porte (fig. 23). Ce dernier arrangement a conduit à la destruction des monuments funéraires les plus proches de la voie nord-sud ${ }^{34}$. Seul le mausolée MSL60137 est préservé.

Fig. 23. Le complexe impérial et les monuments funéraires détruits.

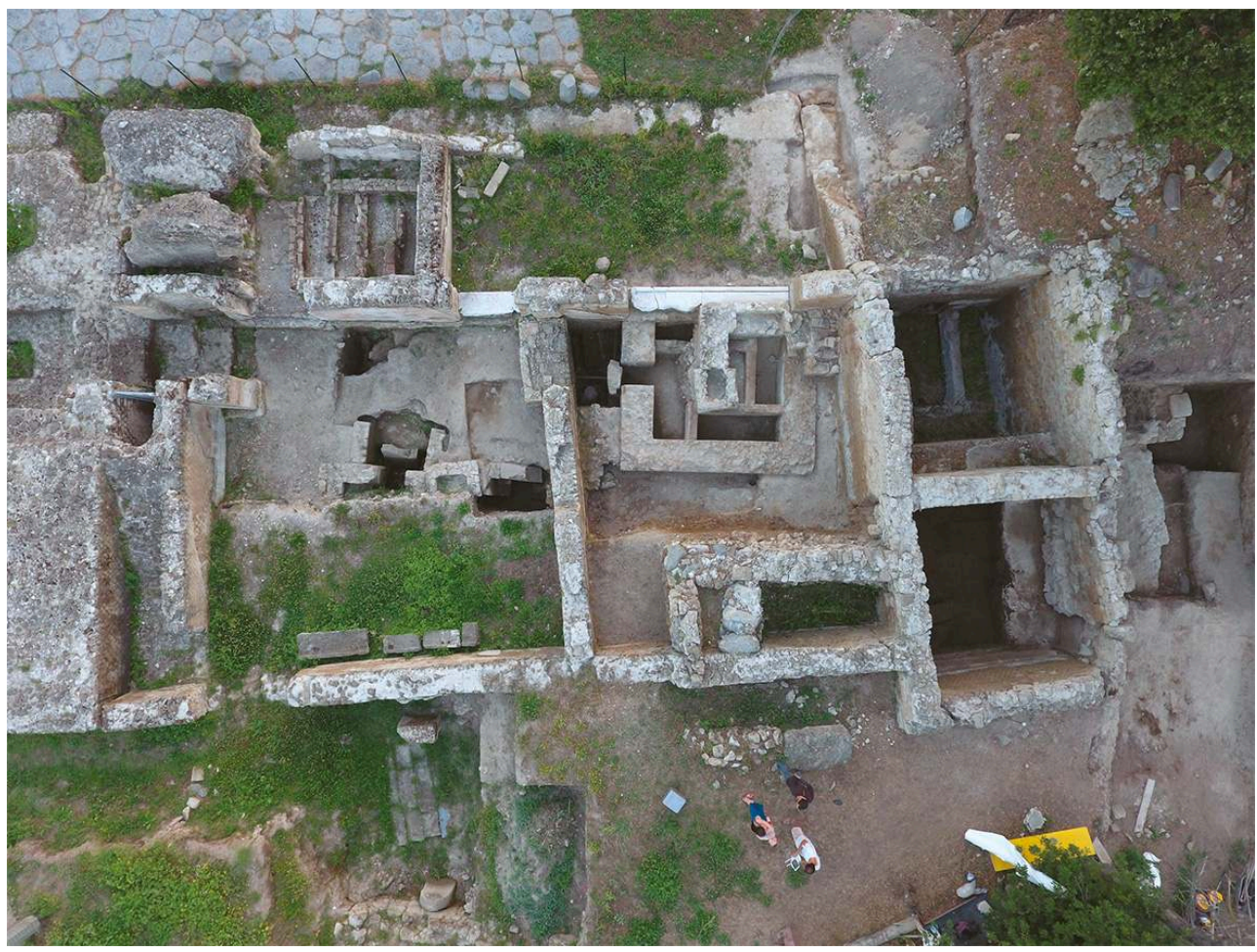

E. Botte, CNRS, CCJ / (c) CC BY-NC-ND.

\section{BIBLIOGRAPHIE}

BOTTE et al. 2011

Emmanuel Botte, Jean-Pierre Brun, Laetitia Cavassa, Gianluca D'Avino, Nicola Meluziis, Priscilla Munzi, Guilhem Chapelin, Anselme Cormier, Milena Costagliola, Serena D’Onofrio, Frédéric Girot, Aline Lacombe, Stéphanie Le Berre, Laure Métais, Ophelie Vauxion et Sophie Girardot, «Cumes », 
Mélanges de l'École française de Rome - Antiquité, en ligne, http://journals.openedition.org/mefra/ 515, consulté le 26 janvier 2021.

BRUN et al. 2012

Jean-Pierre Brun, Priscilla Munzi, Laetitia Cavassa, Stéphanie Le Berre, Nicola Meluziis, Dorothée Neyme, «Cumes », Chronique des activités archéologiques de l'École française de Rome, Italie du Sud, en ligne, http://cefr.revues.org/633, consulté le 26 janvier 2021.

BRUN et al. 2014

Jean-Pierre Brun, Priscilla Munzi, Laetitia Cavassa, Guilhem Chapelin, Pauline Duneufjardin, Stéphanie Le Berre, Stéphanie Mailleur, Nicola Meluziis, Dorothée Neyme, John-Marc Piffeteau et Arnaud Watel, «Cumes ", Chronique des activités archéologiques de l'École française de Rome, Italie du Sud, en ligne, http://cefr.revues.org/1076, consulté le 20 janvier 2021.

BRUN et al. 2017

Jean-Pierre Brun, Priscilla Munzi, Guilhem Chapelin, Marina Covolan, Bastien Lemaire, Marcella Leone, Nicola Meluziis et Géraldine Sachau-Carcel, « Recherches archéologiques dans la nécropole de la Porte médiane à Cumes ", Chronique des activités archéologiques de l'École française de Rome, Italie du Sud, en ligne, http://journals.openedition.org/cefr/1786, consulté le 20 janvier 2021.

BRUN et al. 2019

Jean-Pierre Brun, Priscilla Munzi, Guilhem Chapelin, Marina Covolan, Bastien Lemaire, Marcella Leone et Géraldine Sachau-Carcel, «Recherches archéologiques dans la nécropole de la Porte médiane à Cumes. Rapport d'activité 2017-2018 (première partie) »Chronique des activités archéologiques de l'École française de Rome, Italie du Sud, en ligne, http://journals.openedition.org/ cefr/232, consulté le 20 janvier 2021.

BRUN, MUNZI et al. sous presse

Jean-Pierre Brun, Priscilla Munzi, Elisa Conca, Chiara Germinario, Celestino Grifa, Marcella Leone, Dorothée Neyme, Serena Sechi, « Un banchetto per l'eternità. Nuove scoperte dalla necropoli di Cuma », Pareti dipinte. Dallo scavo alla valorizzazione. Atti del XIV Colloquio AIPMA - Association Internationale pour la Peinture Murale Antique (Napoli-Ercolano, 9-13 Settembre 2019), sous presse. BRUN, MUNZI, POUZADOUX 2017

Jean-Pierre Brun, Priscilla Munzi, Claude Pouzadoux, « 'Memorie dal sottosuolo': una nuova tomba 'a schiena' di età sannita nella necropoli di Cuma ", in Luigi Cicala, Bianca Ferrara (éd.), Kithon Lydios. Studi di storia e archeologia con Giovanna Greco, Naples, Naus, Quaderni del Centro Studi Magna Grecia 22, 2017, p. 373-391.

CAMILLI 1999

Andrea Camilli, Ampullae. Balsamari ceramici di età ellenistica e romana, Rome, Fratelli Palombi, 1999.

CAMODECA 2017

Giuseppe Camodeca, " Nuove iscrizioni funerarie latine di epoca tardorepubblicana da Cumae », in Simona Antolini et al. (éd.), Colonie e municipi nell'era digitale: documentazione epigrafica per la conoscenza delle città antiche, Atti del convegno di studi (Macerata, 10-12 dicembre 2015), Tivoli, Edizioni Tored, 2017, p. 47-70.

CAVASSA 2016

Laetitia Cavassa, « Des pyxides en verre à décor peint à l'époque hellénistique (fin IV ${ }^{\mathrm{e}}$-fin $\mathrm{II}^{\mathrm{e}}$ siècle avant J.-C.) », in Journal of glass studies 58, Corning, Corning Museum of Glass, 2016, p. 21-56. 
CAVASSA et al. 2013

Laetitia Cavassa, Jean-Pierre Brun, Nicola Meluziis, Priscilla Munzi, « De Cumes à Vienne, sur les traces d'une pyxide en verre à décor peint (II ${ }^{\mathrm{e}}$ av. J.-C.) », in Bulletin AFAV 2013, http:// www.afaverre.fr/pdf/bull2013/2013-01-cavassa\%20et\%20al.pdf, consulté le 26 janvier 2021, p. 9-14.

CAVASSA, LEONE, MUNZI 2016

Laetitia Cavassa, Marcella Leone, Priscilla Munzi, « Le repos des cumains. Typochronologie des vases ossuaires ( $\mathrm{I}^{\mathrm{er}}$ siècle av. J.-C. - $\mathrm{I}^{\mathrm{er}}$ siècle apr. J.-C.) ", in SFECAG 2016, Actes du Congrès international d'Autun (Saône-et-Loire, 25-28 mai 2016), Marseille, SFECAG, p. 257-268.

COLIVICCHI 2001

Fabio Colivicchi, Catalogo del Museo Nazionale di Taranto III, 2. Alabastra tardo-ellenistici e romani dalla necropoli di Taranto, Tarante, Scorpione editrice, 2001.

COLIVICCHI 2002

Fabio Colivicchi, La necropoli di Ancona (IV-I secolo a.C.). Una comunità italica fra ellenismo e romanizzazione, Naples, Loffredo editore, 2002.

D'AGOSTINO, D'ANDREA 2002

Bruno d'Agostino, Andrea D'Andrea (éd.), Cuma. Nuove forme d'intervento per lo studio del sito antico, Naples, Istituto Universitario Orientale, AION Quad. 14, 2002.

D'AGOSTINO, FRATTA, MALPEDE 2005

Bruno d'Agostino, Francesca Fratta, Valentina Malpede (éd.), Cuma. Le fortificazioni I. Lo scavo 1994-2002, Naples, Istituto Universitario Orientale, AION Quad. 15, 2005.

D'AGOSTINO, GIGLIO 2012

Bruno d'Agostino, Marco Giglio (éd.), Cuma. Le fortificazioni 3. Lo scavo 2004-2006, Naples, Direzione regionale per i Beni culturali e paesaggistici della Campania, 2012.

DE JORIO 1824

Andrea de Jorio, Metodo per rinvenire e frugare i sepolcri degli antichi, Naples, Stamperia della Società Filomatica, 1824.

DE TOMMASO 1990

Giandomenico De Tommaso, Ampullae vitreae: contenitori in vetro di unguenti e sostanze aromatiche dell'Italia romana, I sec. a.C. - III sec. d.C., Rome, G. Bretschneider, Archaeologica 94, 1990.

GABRICI 1913

Ettore Gabrici, Cuma, Rome, Reale Accademia dei Lincei, Monumenti Antichi LXXII, 1913.

IASIELLO 2017

Italo Iasiello, Napoli da capitale a periferia. Archeologia e mercato antiquario in Campania nella seconda metà dell'Ottocento, Naples, FedOa, 2017.

LEONE sous presse

Marcella Leone, « A perfume for eternity? Unguentaria from the necropolis of Cumae in Magna Graecia (the end of the second century BC -first half of the first century AD) », in Ergün Lafli et al. (éd.), Unguentarium. A terracotta vessel form in the Hellenistic, Roman and early Byzantine Mediterranean, International symposium (Izmir, 17-18 maggio 2018), sous presse.

MUNZI 2019

Priscilla Munzi, « Forme di autorappresentazione nella necropoli osca di Cuma tra il II e il I secolo a.C. », in Marina Cipriani et al. (éd.), Dialoghi sull'Archeologia della Magna Grecia e del 
Mediterraneo III.1, Atti del $3^{\circ}$ Convegno Internazionale di Studi (Paestum, 16-18 novembre 2018), Paestum, Pandemos, 2019, p. 109-122.

MUNZI sous presse

Priscilla Munzi, «Su alcune tombe a camera ipogea e volta a botte delle necropoli settentrionali di Cuma », in Carmelo Malacrino, Simonetta Bonomi (éd.) Ollus leto datus est. Architettura, topografia e rituali funerari nelle necropoli dell'Italia meridionale e della Sicilia fra antichità e medioevo, Atti del Convegno Internazionale di Studi (Reggio Calabria,-22-25 ottobre 2013), sous presse.

MUNZI et al. 2018

Priscilla Munzi, Jean-Pierre Brun, Giuseppe Camodeca, Henry Duday, Marcella Leone, « All'ombra de' cipressi e dentro l'urne... . La latinizzazione della necropoli cumana », in Valentino Nizzo (éd.), Archeologia e Antropologia della Morte, III Incontro di studi di Archeologia e Antropologia a confronto, Atti dell'incontro internazionale (Roma, 20-22 maggio 2015), Rome, Editorial Service System, 2018, p. 101-127.

RESCIGNO 2008

Carlo Rescigno, « La necropoli di epoca sannitica e la pittura funeraria », in Fausto Zevi et al. (éd.), Museo Archeologico dei Campi Flegrei. Catalogo generale. Cuma 1, Naples, Electa, 2008, p. 264-265.

STEVENS 1883

Emilio Stevens, "Cuma », in Notizie degli scavi di Antichità, Rome, Accademia Nazionale dei Lincei, 1883, p. 270-284.

VALENZA MELE 1990

Nazarena Valenza Mele, "La necropoli di Cuma: il superamento della comunità primitiva ", in Marcello Tagliente (éd.), Italici in Magna Grecia. Lingua, insediamenti e strutture, Atti del Convegno (Acquasparta, 30-31 maggio 1986), Venosa, Osanna Edizioni, 1990, p. 23-33.

VALENZA MELE 2010

Nazarena Valenza Mele, « Appendice. Le tombe di epoca arcaica e classica nel contesto della necropoli cumana: appunti topografici », in VALENZA MELE, RESCIGNO 2010, p. 206-210.

VALENZA MELE, RESCIGNO 2010

Nazarena Valenza Mele, Carlo Rescigno (éd.), Cuma. Studi sulla necropoli: scavi Stevens 1878-1896, Rome, « L'Erma » di Bretschneider, Sup. Archeologia Classica 6, 2010.

\section{NOTES}

1. Sur le fossé identifié à l'extérieur des remparts nord, pour le moment exclusivement au moyen de carottages géo-archéologiques, voir Amato, Guastaferro, Lupia dans D'AGOSTINO, D 'ANDREA 2002, p. 95, 97, 100-102, tableaux 7, 8, coupes 1 , 2; sur la problématique du fossé, voir B. d'Agostino dans D'AGostino, D'ANDREA 2002, 16. Voir aussi F. Fratta dans D'AGOSTINO, FRATTA, MALPEDE 2005, p. 39-42.

2. Sur les interventions réalisées sur les murs et sur la structure de la Porte médiane entre le $\mathrm{I}^{\mathrm{er}}$ av. J.-C. et le $\mathrm{II}^{\mathrm{e}} \mathrm{s}$. apr. J.-C., voir V. Malpede dans D'Agostino, FRATTA, MALPEDE 2005, p. 61-65.

3. BRUN et al. 2019.

4. Ce compte-rendu inclus aussi la zone E72, fouillée en 2017 et qui a permis de mettre au jour un tombeau à chambre hypogée et une série de tombes d'époque hellénistique qui n'ont pas été présentées dans Brun et al. 2019. En 2020, la crise sanitaire a conduit à 
réduire la durée, les effectifs et les objectifs de la campagne de terrain. La mission prévue à Cumes a été réalisée en juillet et elle n'a rassemblé que quatre archéologues durant deux semaines, ainsi que des ouvriers et des engins mécaniques pour des travaux d'aménagement de l'aire de la nécropole (22 juin-3 juillet).

5. Les études consacrées à la nécropole de Cumes après la conquête samnite sont nombreuses. En ce qui concerne la typologie des sépultures, outre les pages de E. Stevens dans les Notizie degli Scavi de 1883 (STEVENS 1883, p. 270-284), on note également les textes de E. Gabrici dans son volume de 1913 consacré à Cumes (GABRICI 1913). L'article de N. Valenza Mele de 1990 pour la phase des IV-III ${ }^{\mathrm{e}}$ s. (VALENZA MELE 1990, p. 23-33), et le volume qu'elle a édité avec C. Rescigno sur les fouilles Stevens entre 1878 et 1896, bien que principalement consacré à la nécropole de Cumes aux périodes archaïque et classique, restent fondamentaux (VALENZA MELE, RESCIGNO 2010).

6. STEVENS 1883, p. 274-275, pl. IV ; GABRICI 1913, col. 671-672.

7. Le profil de la section du côté court nord-est/sud-ouest rappelle le type D dans STEVENS 1883, pl. IV.

8. Triens en bronze avec Rome/proue-épi de blé, émise par un atelier Sicilien, 211-210 av. J.-C. (RRC 72/6).

9. Le profil de la section du long côté est-ouest est assimilé au type E dans STEVENS 1883, pl. IV.

10. As de bronze avec Janus/proue datable entre la fin du III et le début du $\mathrm{II}^{\mathrm{e}}$ s. av. J.-C.

11. BRUN, MUNZI, POUZADOUX 2017.

12. RESCIGNO 2008, p. 264.

13. VALENZA MELE 1990.

14. Sur ces phases : MUNZI 2019, p. 109-122.

15. MUNZI 2019, p. 112-113; pour une édition plus complète du contexte : BRUN, MUNZI et al. sous presse.

16. Sur la société de fouille "Tishkiewicz, Bovet et de Gibot» voir IASIELLO 2017, p. 169-175.

17. Munzi 2019, p. 111-114.

18. MUNZI et al. 2018, p. 101-127.

19. Cfr. supra.

20. вотTE et al. 2011.

21. BOTTE et al. 2011 ; CAVASSA et al. 2013 ; MUNZI sous presse.

22. BRUN et al. 2014. La tombe MSL29027 a été pillée à diverses reprises. Le monument est semi-enterré, bâti en opus incertum et avec un toit à double pente recouvert de mortier de tuileau. La façade dégradée par les pillages, comportait trois cippes de tuf. L'un manque, l'autre porte une décoration géométrique et le troisième une inscription très incomplète. La chambre funéraire est couverte d'une voûte ogivale construite en moellons de tuf quadrangulaires disposés de chant selon un schéma radial; on y pénétrait par une porte désaxée au sommet arrondi et elle comportait seulement un lit. Dans une seconde phase, le monument semble avoir subi une rénovation avec un doublement des murs périmétraux en opus quasi reticulatum, des chaînages d'angle en moellons de tuf de forme rectangulaire. 
23. BRUN et al. 2013.

24. BRUN et al. 2018.

25. BOTTE et al. 2011.

26. BRUN et al. 2014.

27. BRUN et al. 2013.

28. BRUN et al. 2019.

29. BRUN et al. 2017.

30. BRUN et al. 2019.

31. Sur les recherches de l'Università L'Orientale di Napoli sur les fortifications dans le secteur de la Porte médiane: D’Agostino, FrattA, MALPEDE 2005 ; D’Agostino, Giglio 2012. Sur le fossé défensif, voir supra, note 1.

32. Sur ces aspects, voir F. Fratta dans D'AGOSTINO, D'ANDREA 2002, p.57, note 182 ; B. d'Agostino dans Cuma. Le fortificazioni 1, Lo scavo 1994-2002, NAPLES 2005, p. 8, note 9. Le collecteur (CN74016) a été reconstruit dans les années 1950 et les interventions sont encore clairement visibles dans la section récemment mise au jour. La couverture, faite de ciment, repose sur des murs en moellons de tuf irréguliers, construits à l'aide d'un coffrage.

33. V. Malpede dans D'AGostino, FRATTA, MALPEDE 2005, p. 61-62.

34. BRUN et al. 2019.

\section{INDEX}

Thèmes : $\mathrm{CJB}$

chronologie https://ark.frantiq.fr/ark:/26678/pcrtZTmusVUU24

lieux https://ark.frantiq.fr/ark:/26678/pcrt4dGhLcuicY

Année de l'opération : 2017, 2018, 2019, 2020

sujets https://ark.frantiq.fr/ark:/26678/pcrtsIm3RuNMGu, https://ark.frantiq.fr/ark:/26678/ pcrt795b632nWw, https://ark.frantiq.fr/ark:/26678/pcrtbza1yQv2Rr, https://ark.frantiq.fr/ark:/ 26678/pcrtJdl7n3HRdk, https://ark.frantiq.fr/ark:/26678/pcrtGdotYbyIR9

peuples https://ark.frantiq.fr/ark:/26678/pcrtS8JHDKUmqH, https://ark.frantiq.fr/ark:/26678/ pcrtkERiWybjc4

\section{AUTEURS}

\section{JEAN-PIERRE BRUN}

Collège de France

PRISCILLA MUNZI

CNRS, Centre Jean Bérard (USR 3133 CNRS-EFR) 


\section{MARINA COVOLAN}

Università degli studi di Salerno

BASTIEN LEMAIRE

Université Paul-Valéry Montpellier 3, UMR 5140 Archéologie des Sociétés Méditerranéennes

MARCELLA LEONE

Centre Jean Bérard (USR 3133, CNRS-EFR)

\section{GÉRALDINE SACHAU-CARCEL}

Université Paul-Valéry Montpellier 3, UMR 5140 Archéologie des Sociétés Méditerranéennes 